\title{
Optical DQPSK Modulation Performance Evaluation
}

\author{
Nelson Costa and Adolfo Cartaxo \\ Group of Research on Optical Fiber Telecommunication Systems, \\ Instituto de Telecomunicações, \\ Dept. of Electrical and Computer Engineering, Instituto Superior Técnico \\ Portugal
}

\section{Introduction}

The always increasing amount of internet traffic in optical networks led to the need of using efficient modulation formats. Conventional on-off-keyed (OOK) signals have been extensively employed in optical communication systems. However, OOK modulation is inadequate for transmission of $40 \mathrm{Gbit} / \mathrm{s}$ per channel, or higher, bit rates (Winzer \& Essiambre, 2006) mainly because of its reduced robustness to fiber nonlinearity, chromatic dispersion and optical filtering at such bit rates. In order to overcome such impairments, several advanced modulation formats received particular attention in the last few years. Some of these modulation formats still carry the information in the amplitude of the signal. However, they also modulate the phase of the signal to increase its robustness to transmission impairments. Some examples of such modulation formats are duobinary and alternate mark inversion.

The most promising modulation formats for future optical networks make use of the phase of the signals to carry information. Among such formats, differential phase-shift-keying (DPSK) and differential quadrature phase-shift-keying (DQPSK) are the ones more often referred. These modulation formats led already to several landmark experimental results confirming their potential (Ho, 2005), (Winzer et al., 2008). The main advantages of DPSK are approximately $3 \mathrm{~dB}$ improvement on optical signal-to-noise ratio (OSNR) when compared with conventional OOK, and improved dispersion and polarization mode dispersion tolerance ( $\mathrm{Xu}$ et al., 2004). DQPSK shows also improved spectral efficiency (Morita \& Yoshikane, 2005).

The evaluation of DQPSK system performance is usually performed using Monte-Carlo (MC) simulation or Karhunen-Loève series expansion (Bosco \& Poggiolini, 2006). The complexity of evaluating the performance of DQPSK system where noise added by optical amplifiers is the main noise source is the main reason for using these performance evaluation methods. However, while MC simulation has the time consumption disadvantage, Karhunen-Loève series expansion derivation is usually of complex nature when rigorous DQPSK system performance evaluation is the goal. Furthermore, these methods provide reduced insight on the effects impairing the transmission system performance unless extensive analysis of different sets of parameters is performed.

Source: Advances in Lasers and Electro Optics, Book edited by: Nelson Costa and Adolfo Cartaxo, ISBN 978-953-307-088-9, pp. 838, April 2010, INTECH, Croatia, downloaded from SCIYO.COM 
Transmission system optimization using such methods is also much time consuming. Thus, much faster, but still accurate, methods for performance evaluation are highly desirable. With this objective, the Gaussian approximation (GA) for the probability density function (PDF) of current at the decision circuit input was investigated. This approximation has been extensively used in OOK modulated system performance evaluation, leading to quite good accuracy (Rebola \& Cartaxo, 2001). However, its use leads to high discrepancies in DQPSK system performance evaluation (Bosco \& Poggiolini, 2006). In order to overcome such limitation, the GA for the phase difference between consecutive symbols was proposed for DPSK system (Xu et al., 2004). This approximation was generalized in (Nelson \& Cartaxo, 2007) in order to take into account the influence of the electrical filter of RX on the phase difference between consecutive symbols, leading to the proposal of an "equivalent" differential phase (EDP). Quite good accuracy on the performance evaluation of DPSK system was achieved in (Nelson \& Cartaxo, 2007).

In this chapter, the use of the GA for the EDP of DQPSK system is analysed. The chapter is organized as follows. In section 2, the DQPSK transmitter (TX) and receiver (RX) structures are analyzed. In section 3, the PDF of the EDP is analysed. In section 4, the mean and standard deviation (STD) of the EDP are derived as a function of the received signal and power spectral density (PSD) of optical noise at RX input and are compared with the ones obtained from MC simulation. In section 5, a semi-analytical simulation method (SASM) for DQPSK system performance evaluation based on the GA for the EDP is proposed. In section 6, the DQPSK system performance estimates obtained with MC simulation are compared with those obtained by the SASM, with the objective of validating the SASM. In section 7, the main conclusions and future research work are outlined.

\section{DQPSK transmitter and receiver structures}

The DQPSK TX and RX are presented in this section. Firstly, the TX structure and operation are briefly discussed. Then, the RX structure and operation are described. Finally, the mathematical description of the RX operation is presented.

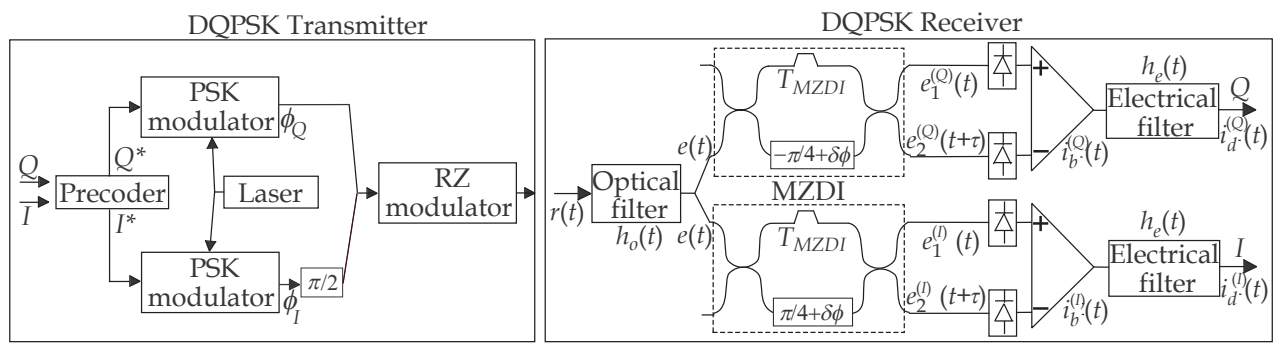

Fig. 1. Scheme of the DQPSK transmitter and receiver.

\subsection{DQPSK transmitter}

Fig. 1 shows the scheme of the DQPSK TX and RX (Ho, 2005). The input data is firstly precoded at the TX. The logic operations of the DQPSK precoder presented in (Ho, 2005) are considered in this chapter. The precoder eliminates error propagating at RX (Ho, 2005). Furthermore, the precoder allows also direct mapping of the bit sequence of each DQPSK component from the input to the output (Bosco \& Poggiolini, 2006). Assuming that the first 
bit is transmitted in the in-phase (I) component and the second bit is transmitted in the quadrature $(\mathrm{Q})$ component of the DQPSK signal, direct mapping is achieved by coding the input data $(I$ and $Q)$ 11, 01, 00 and 10 with the phase differences of $0, \pi / 2, \pi$ and $-\pi / 2$, respectively (Ho, 2005). The coded data $\left(I^{*}\right.$ and $\left.Q^{*}\right)$ is firstly modulated by phase modulators. Then, a $\pi / 2$ phase shift is applied to the I component of the DQPSK signal. The combination of the I and Q components generates the DQPSK signal. Table 1 shows an example of the logical value and phase of the DQPSK signal at several points of the TX.

\begin{tabular}{|c|c|c|c|c|c|c|c|c|c|c|c|c|c|c|c|c|}
\hline$I$ & 1 & 1 & 1 & 1 & 0 & 0 & 0 & 1 & 0 & 0 & 1 & 0 & 1 & 1 & 0 & 0 \\
\hline$Q$ & 1 & 0 & 1 & 0 & 1 & 1 & 1 & 1 & 0 & 0 & 0 & 0 & 1 & 0 & 1 & 0 \\
\hline$I^{*}$ & 0 & 1 & 1 & 1 & 1 & 0 & 0 & 0 & 1 & 0 & 0 & 1 & 1 & 0 & 1 & 0 \\
\hline$Q^{*}$ & 0 & 0 & 0 & 1 & 0 & 0 & 1 & 1 & 0 & 1 & 0 & 1 & 1 & 1 & 1 & 0 \\
\hline$\phi_{I}$ & 0 & $\pi$ & $\pi$ & $\pi$ & $\pi$ & 0 & 0 & 0 & $\pi$ & 0 & 0 & $\pi$ & $\pi$ & 0 & $\pi$ & 0 \\
\hline$\phi_{Q}$ & 0 & 0 & 0 & $\pi$ & 0 & 0 & $\pi$ & $\pi$ & 0 & $\pi$ & 0 & $\pi$ & $\pi$ & $\pi$ & $\pi$ & 0 \\
\hline $\begin{array}{c}\text { Phase at } \\
\text { TX output }\end{array}$ & $\frac{\pi}{4}$ & $-\frac{\pi}{4}$ & $-\frac{\pi}{4}$ & $-\frac{3 \pi}{4}$ & $-\frac{\pi}{4}$ & $\frac{\pi}{4}$ & $\frac{3 \pi}{4}$ & $\frac{3 \pi}{4}$ & $-\frac{\pi}{4}$ & $\frac{3 \pi}{4}$ & $\frac{\pi}{4}$ & $-\frac{3 \pi}{4}$ & $-\frac{3 \pi}{4}$ & $\frac{3 \pi}{4}$ & $-\frac{3 \pi}{4}$ & $\frac{\pi}{4}$ \\
\hline $\begin{array}{c}\text { Phase } \\
\text { difference }\end{array}$ & 0 & $-\frac{\pi}{2}$ & 0 & $-\frac{\pi}{2}$ & $\frac{\pi}{2}$ & $\frac{\pi}{2}$ & $\frac{\pi}{2}$ & 0 & $\pi$ & $\pi$ & $-\frac{\pi}{2}$ & $\pi$ & 0 & $-\frac{\pi}{2}$ & $\frac{\pi}{2}$ & $\pi$ \\
\hline
\end{tabular}

Table 1. Logical value and phase of the DQPSK signal at several points of the TX.

The DQPSK signal at the return-to-zero (RZ) modulator input is a non-return-to-zero (NRZ)-DQPSK signal. The RZ modulator is used for generating a RZ-DQPSK signal, if that is the goal. Other TX schemes for generating the DQPSK signals are referred in (Ho, 2005), such as the cascading of two phase modulators or the driving of a single phase modulator by a four level signal. However, the TX scheme shown in Fig. 1 is the one more often referred in the literature mainly because of its simpler implementation. Thus, only the TX scheme shown in Fig. 1 is considered in this chapter. Moreover, as the optical intensity is ideally a constant pulse train without ripple between symbols after the RZ modulator, independently of the TX structure (Ho, 2005), the results shown in this chapter are similar for other TX schemes.

Fig. 2 shows the optical intensity at the output of an ideal TX in case of transmitting a 33\% duty-cycle RZ-DQPSK signal or a NRZ-DQPSK signal considering the symbol sequence shown in Table 1. The same average power is imposed for both signals. Fig. 2 shows that the shape of optical intensity is independent of the transmitted symbol sequence.

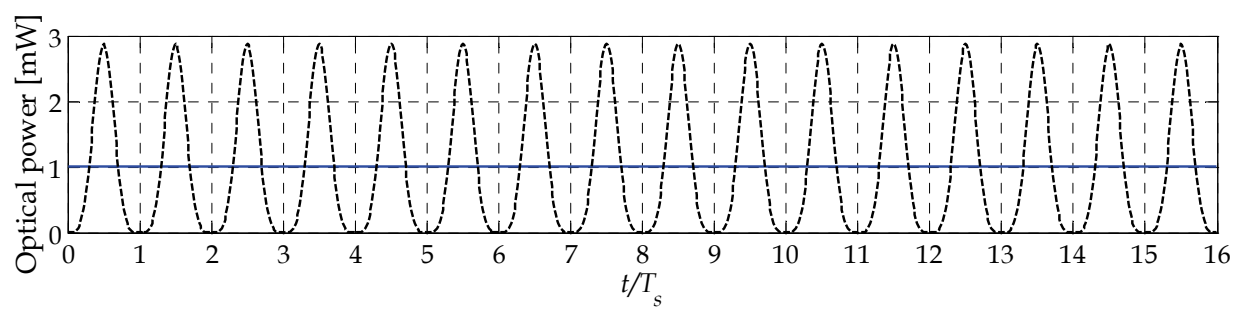

Fig. 2. Optical intensity at the TX output obtained by simulation. Continuous line: NRZDQPSK signal; dashed line: 33\% duty-cycle RZ-DQPSK signal. 


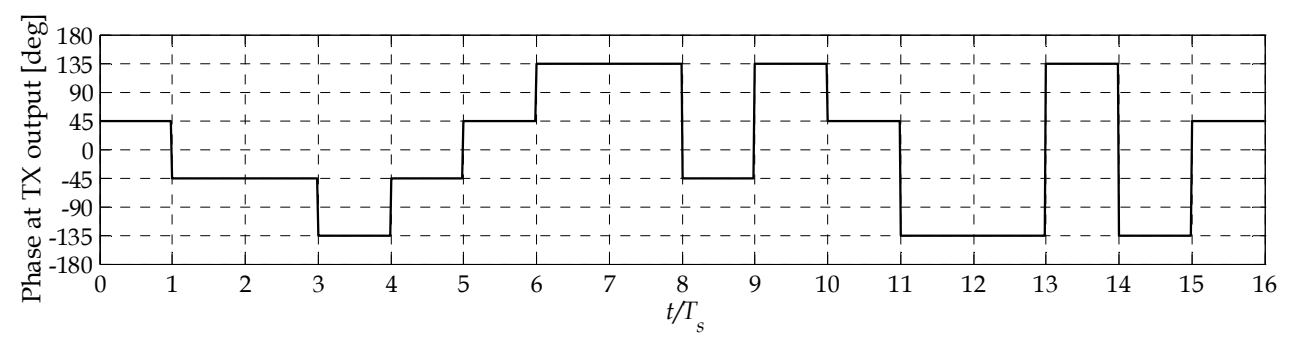

Fig. 3. Phase of the DQPSK signal at the TX output obtained by simulation.

Fig. 3 shows the phase at the output of an ideal TX. Fig. 3 shows that the phase changes accordingly to the symbol sequence, being constant for the duration of each symbol. The shape of the phase is independent of the duty-cycle of the DQPSK signal.
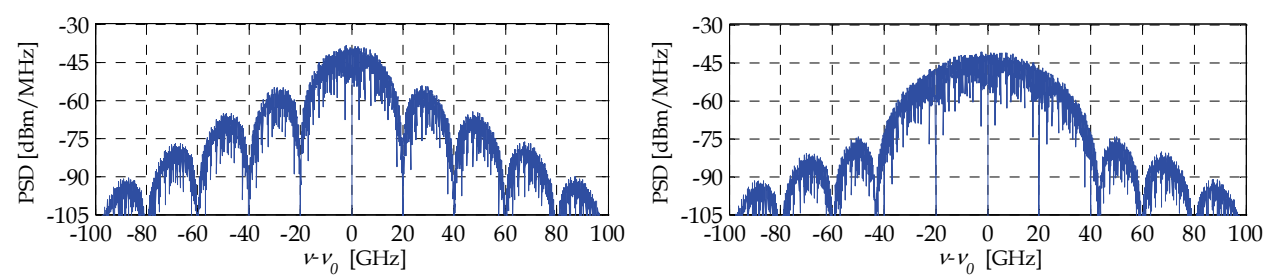

Fig. 4. PSD of the optical field at the output of an ideal TX obtained by simulation. Left: NRZ-DQPSK signal; right: 33\% duty-cycle RZ-DQPSK signal.

Fig. 4 shows the PSD of the optical field at the TX output. As a symbol rate of $20 \mathrm{Gbaud} / \mathrm{s}$ is imposed, the first null, in case of the NRZ-DQPSK signal, occurs for $v-v_{0}=20 \mathrm{GHz}$, where $v$ is the optical frequency and $v_{0}$ is the optical carrier frequency. In case of the $33 \%$ dutycycle RZ-DQPSK signal, a much wider signal spectrum is observed, as expected. A detailed discussion on the spectral characteristics of RZ signals can be found in (Ip \& Kahn, 2006).

\subsection{DQPSK receiver}

At the RX side, the DQPSK signal is firstly filtered by an optical filter. Then, the resulting signal is split to a pair of Mach-Zehnder delay interferometers (MZDIs) to perform the detection of the I and Q components of the DQPSK signal. Both output ports of each MZDI carry the full information. Indeed, the signal at the output of the two ports of each MZDI is logically conjugated. Thus, the detection of the output of a single port of the MZDI is sufficient to recover the information. However, the use of a balanced detector allows improving the OSNR by about $3 \mathrm{~dB}$ and, therefore, the use of the balanced detector is usually considered. The electrical current at the decision circuit input results from filtering the electrical current at the balanced detector output.

Assuming an ideal DQPSK RX, neglecting the filtering impact and defining the received signal as $E_{r}(t)=A e^{j \phi(t)}$, where $A$ and $\phi(t)$ are the amplitude and phase of the received signal, respectively, the electrical current at the decision circuit input is $i_{d}^{(I, Q)}(t)=A^{2} / 2 \cos \left(\phi(t)-\phi\left(t-T_{s}\right)+\theta\right)$, where $T_{s}$ is the symbol duration and $\theta=\pi / 4$ for the I component of the DQPSK signal, and $\theta=-\pi / 4$ for the Q component of the DQPSK signal. 
This expression shows that the combined effect of the MZDI and balanced detector translates in creating dependence of the received electrical current on the phase difference between two consecutive symbols. Thus, the transmitted information can be extracted from the EDP, $\Delta \phi_{e}^{(I, Q)}(t)$, defined as $\Delta \phi_{e}^{(I, Q)}(t)=\phi(t)-\phi\left(t-T_{s}\right)+\theta$.

\begin{tabular}{|c|c|c|c|c|c|c|c|c|c|c|c|c|c|c|c|c|}
\hline $\begin{array}{c}\text { Phase } \\
\text { difference }\end{array}$ & 0 & $-\frac{\pi}{2}$ & 0 & $-\frac{\pi}{2}$ & $\frac{\pi}{2}$ & $\frac{\pi}{2}$ & $\frac{\pi}{2}$ & 0 & $\pi$ & $\pi$ & $-\frac{\pi}{2}$ & $\pi$ & 0 & $-\frac{\pi}{2}$ & $\frac{\pi}{2}$ & $\pi$ \\
\hline $\begin{array}{c}\text { EDP } \\
\text { (I comp.) }\end{array}$ & $\frac{\pi}{4}$ & $-\frac{\pi}{4}$ & $\frac{\pi}{4}$ & $-\frac{\pi}{4}$ & $\frac{3 \pi}{4}$ & $\frac{3 \pi}{4}$ & $\frac{3 \pi}{4}$ & $\frac{\pi}{4}$ & $-\frac{3 \pi}{4}$ & $-\frac{3 \pi}{4}$ & $-\frac{\pi}{4}$ & $-\frac{3 \pi}{4}$ & $\frac{\pi}{4}$ & $-\frac{\pi}{4}$ & $\frac{3 \pi}{4}$ & $-\frac{3 \pi}{4}$ \\
\hline$I$ & 1 & 1 & 1 & 1 & 0 & 0 & 0 & 1 & 0 & 0 & 1 & 0 & 1 & 1 & 0 & 0 \\
\hline $\begin{array}{c}\text { EDP } \\
\text { (Q comp.) }\end{array}$ & $-\frac{\pi}{4}$ & $-\frac{3 \pi}{4}$ & $-\frac{\pi}{4}$ & $-\frac{3 \pi}{4}$ & $\frac{\pi}{4}$ & $\frac{\pi}{4}$ & $\frac{\pi}{4}$ & $-\frac{\pi}{4}$ & $\frac{3 \pi}{4}$ & $\frac{3 \pi}{4}$ & $-\frac{3 \pi}{4}$ & $\frac{3 \pi}{4}$ & $-\frac{\pi}{4}$ & $-\frac{3 \pi}{4}$ & $\frac{\pi}{4}$ & $\frac{3 \pi}{4}$ \\
\hline$Q$ & 1 & 0 & 1 & 0 & 1 & 1 & 1 & 1 & 0 & 0 & 0 & 0 & 1 & 0 & 1 & 0 \\
\hline
\end{tabular}

Table 2. EDP of the I and Q components and corresponding bit sequence.
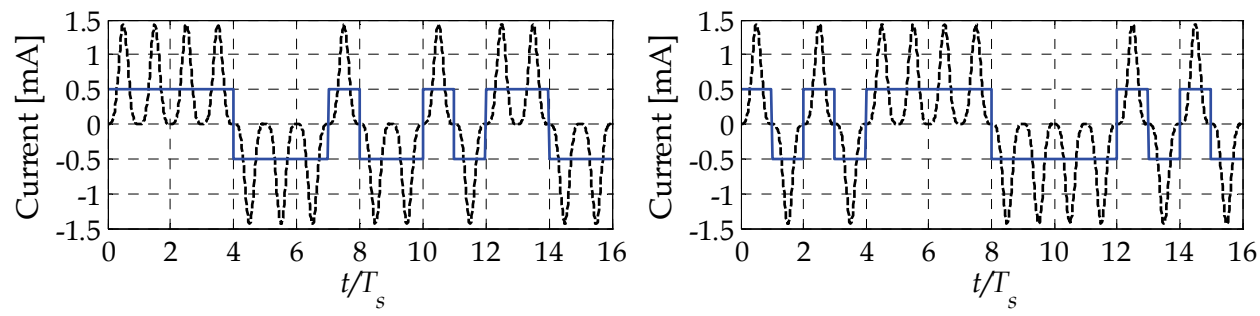

Fig. 5. Electrical current at the decision circuit input in the I component (left) and in the Q component (right) of the DQPSK signal obtained by simulation. Continuous line: NRZDQPSK signal; dashed line: 33\% duty-cycle RZ-DQPSK signal.

Table 2 shows the EDP of the I and Q components after balanced detection for the symbol sequence presented in Table 1 , and the corresponding detected bit sequence. The analysis of Table 2 shows that, for both I and Q components, two EDP give rise to the same bit decision: $\pm \pi / 4$ for the " 1 " bit and $\pm 3 \pi / 4$ for the " 0 " bit. This result is a consequence of the electrical current being given as a function of a cosine function. Thus, to decide correctly, two EDP thresholds levels should be set: one threshold level between the symbols transmitted with nominal means of $-3 \pi / 4$ and $-\pi / 4$ and another threshold level between the symbols transmitted with nominal means of $\pi / 4$ and $3 \pi / 4$. However, the RX performs bit decision on the detected electrical current where there is only one electrical current level threshold. Both EDP threshold levels must correspond to that electrical current threshold level. Thus, as the electrical current is given as a function of a cosine function with constant absolute value, the positive and the negative EDP threshold levels must be symmetric. For example, the electrical current level threshold of 0 corresponds to the EDP threshold level of $\pm \pi / 2$.

Fig. 5 shows the electrical current at the decision circuit input in the I and Q components when the symbol sequence shown in Table 1 is transmitted. The comparison of Fig. 5 with Fig. 2 reveals that the detected signal has only half the amplitude of the signal at the TX output. This is a consequence of splitting the optical signal into the pair of MZDIs at the RX. 


\subsection{Mathematical characterization of DQPSK receiver and of noise}

The mathematical characterization of the RX operation is important for assessing the impact of the RX on the received signal analytically.

The signal field at the optical filter input is assumed to be completely polarized while optical noise is assumed to be unpolarized and additive with known PSD. When no nonlinear fiber transmission is considered, the amplified spontaneous emission (ASE) noise of optical amplifiers is usually the main noise source and is modelled as additive white Gaussian noise (AWGN) at RX input over each polarization direction.

For additive optical noise, the optical field at the RX input is written as $\mathbf{r}(t)=\mathbf{r}_{i n}(t)+\mathbf{r}_{n}(t)$ where $\mathbf{r}_{i n}(t)=r_{i n}(t) \mathbf{u}_{\|}$and $\mathbf{r}_{n}(t)=r_{n, \|}(t) \mathbf{u}_{\|}+r_{n, \perp}(t) \mathbf{u}_{\perp} \cdot \mathbf{r}_{i n}(t)$ and $\mathbf{r}_{n}(t)$ are the Jones vectors of the DQPSK signal and noise fields at the RX input, respectively, and $r_{i n}(t), r_{n, \|}(t)$ and $r_{n, \perp}(t)$ are the DQPSK signal and the noise components, at the RX input, over the parallel (to the signal) and perpendicular polarization directions, respectively, defined by the unit vectors $\mathbf{u}_{\|}$and $\mathbf{u}_{\perp}$. The lowpass equivalent of the DQPSK signal and noise fields, $\mathbf{s}_{\text {in }}(t)$ and $\mathbf{n}_{n}(t)$, respectively, are defined by $\mathbf{r}_{i n}(t)=\sqrt{2} \Re\left(s_{i n}(t) e^{j 2 \pi v_{0} t}\right)=\sqrt{2} \Re\left(s_{i n}(t) e^{j 2 \pi v_{0} t}\right) \mathbf{u}_{\|}$and $\mathrm{r}_{n}(t)=\sqrt{2} \mathfrak{R}\left(\mathrm{n}_{n}(t) e^{j 2 \pi v_{0} t}\right)=\sqrt{2} \mathfrak{R}\left(\left[n_{n, \|}(t) \mathrm{u}_{\|}+n_{n, \perp}(t) \mathrm{u}_{\perp}\right] e^{j 2 \pi v_{0} t}\right)$, where $s_{i n}(t), \quad n_{n, \|}(t) \quad$ and $n_{n, \perp}(t)$ are the lowpass equivalents of $r_{i n}(t), r_{n, \|}(t)$ and $r_{n, \perp}(t)$, respectively, and $\Re\{x\}$ is the real part of $x$.

By assuming that the optical filter is polarization-maintaining with impulse response $h_{0}(t)$, the optical signal at each MZDI input is given by $\mathbf{e}(t)=\mathbf{e}_{s}(t)+\mathbf{e}_{n}(t)$, with $\mathbf{e}_{s}(t)=\mathbf{r}_{i n}(t) * h_{o}(t) / \sqrt{2}$ and $\mathbf{e}_{n}(t)=\mathbf{r}_{n}(t) * h_{o}(t) / \sqrt{2}$. The $\sqrt{2}$ factor quantifies the power splitting into the pair of MZDIs. Ideally, the central frequency of the optical filter, $v_{\mathcal{c}}$, is the same of the optical carrier. However, a frequency detuning may occur. Thus, the impact of frequency detuning, defined as $\Delta v=v_{c}-v_{0}$, on the DQPSK system performance is assessed. Defining the lowpass equivalent of the optical filter impulse response, $h_{o, l}(t)$, as $h_{o}(t)=2 \Re\left(h_{o, l}(t) e^{j 2 \pi v_{0} t}\right)$, the lowpass equivalent of the signal at each MZDI input, $\mathbf{e}_{l}(t)$, defined as $\mathbf{e}(t)=\sqrt{2} \Re\left(\mathbf{e}_{l}(t) e^{j 2 \pi v_{0} t}\right)$, is $\mathbf{e}_{l}(t)=\mathbf{s}_{f}(t)+\mathbf{n}_{f}(t)$ where

$$
\begin{aligned}
& \mathbf{s}_{f}(t)=s_{i n}(t) * h_{o}(t) / \sqrt{2} \mathbf{u}_{\|}=s(t) \mathbf{u}_{\|} \\
& \mathbf{n}_{f}(t)=\left(n_{n, \|}(t) * h_{o}(t) \mathbf{u}_{\|}+n_{n, \perp}(t) * h_{o}(t) \mathbf{u}_{\perp}\right) / \sqrt{2}=n_{\|}(t) \mathbf{u}_{\|}+n_{\perp}(t) \mathbf{u}_{\perp} .
\end{aligned}
$$

The total optical signal over each polarization direction is $\mathbf{e}_{l}(t)=e_{\|}(t) \mathbf{u}_{\|}+e_{\perp}(t) \mathbf{u}_{\perp}$ with

$$
\begin{aligned}
& e_{\|}(t)=s(t)+n_{\|}(t)=s_{r}(t)+n_{\|, r}(t)+j\left(s_{i}(t)+n_{\|, i}(t)\right) \\
& e_{\perp}(t)=n_{\perp}(t)=n_{\perp, r}(t)+j n_{\perp, i}(t)
\end{aligned}
$$

where the indexes $r$ and $i$ refer to the real and imaginary part of the signals, respectively. Polarization-maintaining MZDIs are assumed. These devices are mathematically modelled by (Bosco \& Poggiolini, 2006):

$$
\begin{aligned}
& e_{1,(\|, \perp)}^{(I, Q)}(t)=1 / 2\left(\gamma e_{(\|, \perp)}\left(t-T_{M Z D I}\right)+e_{(\|, \perp)}(t) e^{j \theta}\right) \\
& e_{2,(I,, \perp)}^{(I, Q)}(t)=1 / 2\left(\mathcal{\gamma}_{(\|, \perp)}\left(t-T_{M Z D I}\right)-e_{(\|, \perp)}(t) e^{j \theta}\right)
\end{aligned}
$$


where $\theta$ quantifies the phase rotation performed by the MZDI. Ideally, $\theta=\pi / 4$ for the I component of the DQPSK signal, and $\theta=-\pi / 4$ for the $\mathrm{Q}$ component of the DQPSK signal. However, an imperfect phase rotation with a phase-shifting of $\delta \phi$ from the ideal case may occur. Thus, $\theta= \pm \pi / 4+\delta \phi$. A frequency detuning parameter, $\Delta f$, can be associated with the imperfect phase rotation by $\Delta f / B_{R}=\delta \phi /(4 \pi)$ (Bosco \& Poggiolini, 2006). $B_{R}$ is the bit rate which is related with the symbol duration by $B_{R}=2 / T_{s} . T_{M Z D I}$ is the delay introduced by the MZDI between its two arms. Ideally, $T_{M Z D I}$ is equal to the symbol duration. However, a delay error, defined as $\delta T_{M Z D I}=T_{M Z D I}-T_{S}$ may occur. The $\gamma$ parameter, which is in the range [0,1], allows taking into account the extinction ratio of the MZDI. $\gamma=0$ means that the delayed signal is eliminated at the MZDI while $\gamma=1$ means that the delayed signal suffers from the same loss as the original signal at the MZDI. The extinction ratio of the MZDI, $\varepsilon$, is related with $\gamma$ by $\varepsilon=(1+\gamma)^{2} /(1-\gamma)^{2}$. A balanced detector is used at the output of each MZDI. The electrical current at a positive-intrinsic-negative (PIN) photodetector output is related with the optical field at its input, $\mathbf{f}(t)$, by $i(t)=R|\mathbf{f}(t)|^{2}=R\left(\left|f_{\|}(t)\right|^{2}+\left|f_{\perp}(t)\right|^{2}\right)$, where $R$ is the PIN photodetector responsivity. Thus, the electrical current of each component of the DQPSK signal at the balanced detector output is given by

$$
\begin{aligned}
i_{b}^{(I, Q)}(t) & =R_{1}\left|\mathrm{e}_{1}^{(I, Q)}(t)\right|^{2}-R_{2}\left|\mathrm{e}_{2}^{(I, Q)}(t+\tau)\right|^{2} \\
& =R_{1}\left(\left|e_{1, \|}^{(I, Q)}(t)\right|^{2}+\left|e_{1, \perp}^{(I, Q)}(t)\right|^{2}\right)-R_{2}\left(\left|e_{2, \|}^{(I, Q)}(t+\tau)\right|^{2}+\left|e_{2, \perp}^{(I, Q)}(t+\tau)\right|^{2}\right)
\end{aligned}
$$

where $\tau$ quantifies the time-misalignment between the signals at the input of the balanced detector. The amplitude-imbalance of the balanced detector is assessed by the $k$ factor, defined as $k=\left(R_{1}-R_{2}\right) /\left(R_{1}+R_{2}\right)$. The electrical current resulting from the balanced detector is filtered by a low-pass electrical filter with impulse response $h_{e}(t)$. Thus, the electrical current at the decision circuit input is $i_{d}^{(I, Q)}(t)=i_{b}^{(I, Q)}(t) * h_{e}(t)$. The electrical current at the decision circuit input may also be expressed in a complex representation as

$$
i_{d}^{(I, Q)}(t)=\Re\left(A_{e}^{(I, Q)}(t) e^{j \Delta \phi_{e}^{(I, Q)}(t)}\right)
$$

where $A_{e}^{(I, Q)}$ is the equivalent amplitude of the electrical current. Using eqs. (3), (4) and (5), the EDP can be written as a function of the optical signal at the MZDI input as:

$$
\begin{aligned}
\Delta \phi_{e}^{(I, Q)}(t)=\arg ( & \frac{R_{1}}{2}\left[\mathcal{F}_{||}(t) e_{||}^{*}\left(t-T_{M Z D I}\right) e^{j \theta}+\mathcal{e}_{\perp}(t) e_{\perp}^{*}\left(t-T_{M Z D I}\right) e^{j \theta}\right] * h_{e}(t) \\
& +\frac{R_{1}}{4}\left[\left|\mathcal{H}_{\mid}\left(t-T_{M Z D I}\right)\right|^{2}+\left|e_{||}(t)\right|^{2}+\left|\mathcal{e}_{\perp}\left(t-T_{M Z D I}\right)\right|^{2}+\left|e_{\perp}(t)\right|^{2}\right] * h_{e}(t) \\
& +\frac{R_{2}}{2}\left[\mathcal{H}_{||}(t+\tau) e_{||}^{*}\left(t-T_{M Z D I}+\tau\right) e^{j \theta}+\mathcal{e}_{\perp}(t+\tau) e_{\perp}^{*}\left(t-T_{M Z D I}+\tau\right) e^{j \theta}\right] * h_{e}(t) \\
& \left.-\frac{R_{2}}{4}\left[\left|\mathcal{H}_{||}\left(t-T_{M Z D I}+\tau\right)\right|^{2}+\left|e_{||}(t+\tau)\right|^{2}+\left|\mathcal{e}_{\perp}\left(t-T_{M Z D I}+\tau\right)\right|^{2}+\left|e_{\perp}(t+\tau)\right|^{2}\right] * h_{e}(t)\right)
\end{aligned}
$$


where $e^{*}(t)$ is the complex conjugate of $e(t)$. In case of an ideal RX, where $\Delta v=0, R_{1}=R_{2}$, $T_{M Z D I}=T_{S}, \quad \theta= \pm \pi / 4, \quad \tau=0$ and $\quad \gamma=1$, the EDP is given by $\Delta \phi_{e}^{(I, Q)}(t)=\arg \left(\left[\left(e_{\|}(t) e_{\|}^{*}\left(t-T_{s}\right)+e_{\perp}(t) e_{\perp}^{*}\left(t-T_{s}\right)\right) e^{j \theta}\right] * h_{e}(t)\right)$ which is the result shown in (Costa \& Cartaxo, 2009). Expression (6) shows that the EDP is a nonlinear function of the signal at the MZDI input. Therefore, the rigorous statistical characterization of the EDP may be quite complex. However, approximated solutions may be used in order to get closed-form expressions for the moments of the EDP. The derivation of closed-form expressions for the moments of the EDP is quite important because it allows the assessment of DQPSK system performance in a time-efficient manner.

\section{Probability density function of the equivalent differential phase}

In this section, the PDF of the EDP is assessed. The PDF of the EDP at the time instant leading to higher eye-opening in the absence of noise is approximately Gaussian-distributed when 33\% duty-cycle RZ-DQPSK pulses are considered, even in presence of RX imperfections (Costa \& Cartaxo, 2009b). However, the PDF of the EDP when NRZ-DQPSK pulses are considered has not been assessed yet, as far as the authors are aware of. Thus, this analysis is performed in this section. Unless otherwise stated, an OSNR of $15 \mathrm{~dB}$, measured in a $0.1 \mathrm{~nm}$ bandwidth, is imposed at the RX input. The bit rate is set to $40 \mathrm{Gbit} / \mathrm{s}$. The frequency response of the modulator of the TX is modelled by a five-pole Bessel electrical filter with a $-3 \mathrm{~dB}$ bandwidth equal to the symbol rate. A second-order super-Gaussian optical filter with a $-3 \mathrm{~dB}$ bandwidth of $40 \mathrm{GHz}$ and a five-pole Bessel electrical filter with a $3 \mathrm{~dB}$ bandwidth equal to $18 \mathrm{GHz}$ are used at the RX side. These parameters lead to a bit error probability (BEP) of about $10^{-5}$ in case of ideal RX. Only the PDF of the EDP and eyediagram of the I component of the DQPSK signal are shown because the $Q$ component of the DQPSK signal showed similar results in all considered cases.

Fig. 6 shows the eye-diagram of electrical current at the electrical filter output when the ideal RX and when several different RX imperfections are considered. The corresponding PDF of the EDP at the time instant leading to higher eye-opening in the absence of noise is also shown. Only the PDF of the EDP of the first symbol transmitted with each nominal mean is shown because the PDFs of the EDP of the remaining symbols transmitted with each nominal mean is similar. Both the actual PDF of the EDP (marks) and the GA for the PDF of the EDP estimated from the mean and STD of the actual PDF (lines) are shown. An ideal RX is considered in Fig. 6 a). In this case, the eye-diagram is quite symmetric at the time instant leading to the higher eye-opening. This symmetry occurs for moderate and wide optical filtering. The PDF of the EDP is approximately Gaussian-distributed with nominal means of $\pm \pi / 4$ and $\pm 3 \pi / 4$. The illustration of imperfect phase rotation is shown in Fig. 6 b). Fig. 6 b) shows that an imperfect phase rotation leads to the closure of the eyediagram. Its impact on the PDF of the EDP is, however, just a shift on the nominal means equal to the imperfection of the phase rotation. The STD of the EDP remains the same. The illustration of the time-misalignment of signals at the balanced receiver input is shown in Fig. 6 c). This RX imperfection leads to quite asymmetric eye-diagrams. Nevertheless, the EDP is still approximately Gaussian-distributed. However, a slight underestimation of the PDF of the EDP at the area of interest (where the PDF of the EDP of the symbols transmitted with nominal means of $\pm \pi / 4$ intersects with the PDF of the EDP of the symbols transmitted 

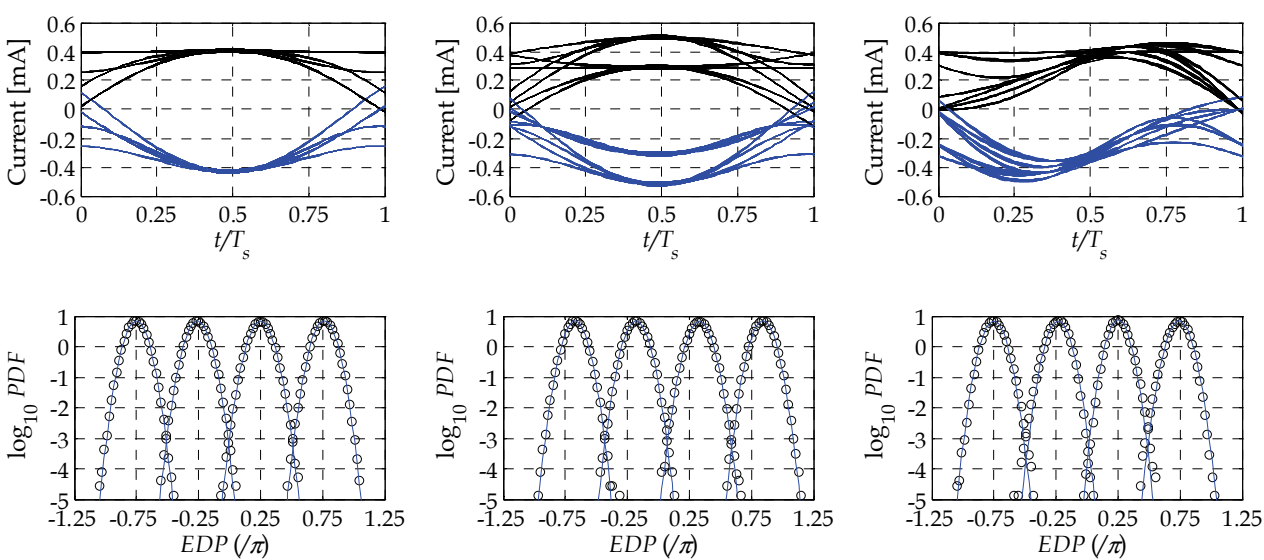

a) Ideal $R X$

b) $\Delta f / B_{R}=2 \%$

c) $\Delta \tau / T_{S}=40 \%$
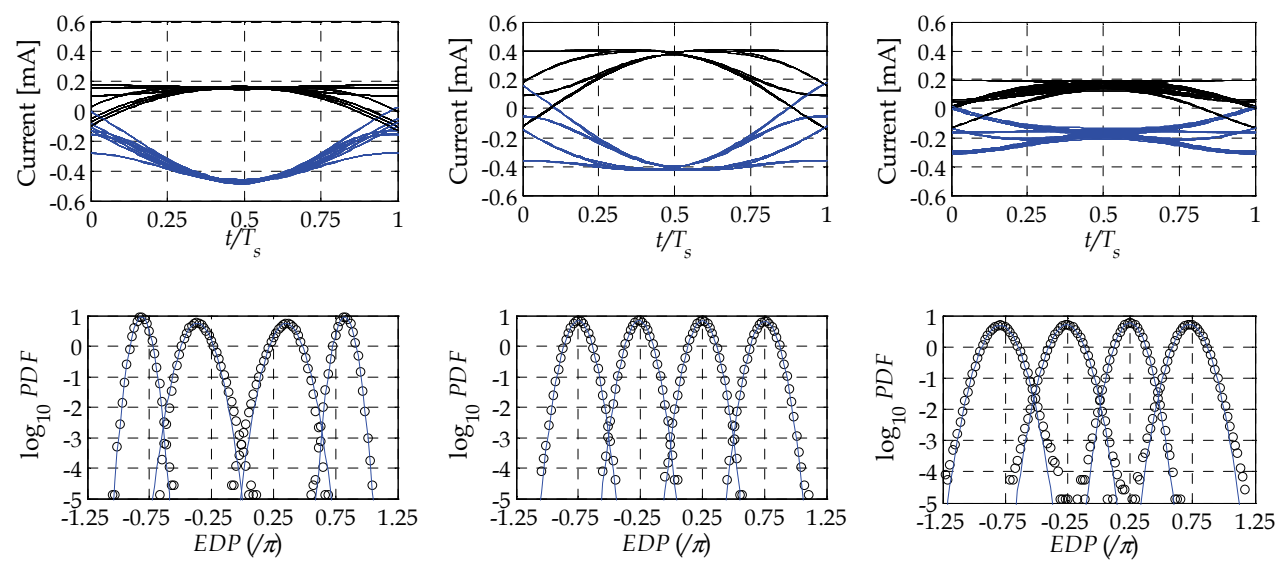

d) $R_{1}=0.5 \mathrm{~A} / \mathrm{W}, R_{2}=1 \mathrm{~A} / \mathrm{W}$

e) $\delta T_{M Z D I} / T_{S}=20 \%$

f) $\Delta v=20 \mathrm{GHz}$

Fig. 6. Eye-diagram of electrical current and corresponding PDF of the EDP in presence of several different RX imperfections. Marks: MC simulation; lines: GA estimated from the results of MC simulation.

with nominal means of $\pm 3 \pi / 4$ ) is performed by the GA. Fig. $6 \mathrm{~d}$ ) illustrates the amplitudeimbalance of detector. This RX imperfection leads to quite asymmetric eye-diagrams and to some inaccuracy in the GA for the PDF of the EDP. However, the EDP at the area of interest is still approximately Gaussian-distributed. The illustration of delay errors of MZDI is shown in Fig. 6 e). This RX imperfection leads to some distortion of the eye-diagram. Nevertheless, the EDP is still approximately Gaussian-distributed. The illustration of the optical filter detuning is shown in Fig. $6 \mathrm{f}$ ). The optical filter detuning leads to considerable degradation of the eye-diagram. The EDP at the area of interest is still approximately Gaussian-distributed. However, the GA tends to slight underestimate the PDF of the EDP. 
Another RX imperfection is the finite extinction ratio of the MZDIs. This imperfection affects only the DQPSK system performance when combined with amplitude-imbalanced detectors (Bosco \& Poggiolini, 2006). In such case, the performance degradation is mainly imposed by the amplitude-imbalance unless much reduced extinction ratios are considered. Thus, both the eye-diagram and PDF of the EDP in presence of finite extinction ratios of the MZDIs are usually similar to those shown in Fig. 6 d).

Fig. 7 shows the eye-diagram of electrical current and the corresponding PDF of the EDP at the decision circuit input when Butterworth electrical filters are considered at the RX side. This analysis allows assessing the impact of the group delay of electrical filters on the eyediagram and PDF of the EDP because the group delay of Butterworth electrical filters is quite different from the one of Bessel electrical filters. The analysis of Fig. 7 shows that the PDF of the EDP remains approximately Gaussian-distributed even when Butterworth electrical filters are considered.
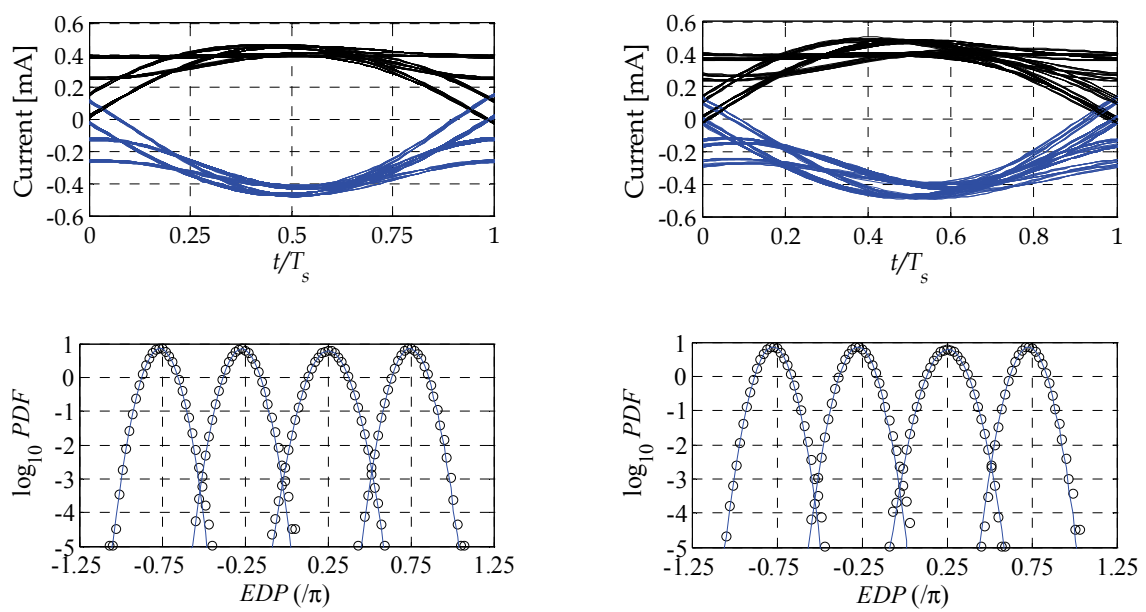

Fig. 7. Eye-diagram of electrical current and corresponding PDF of the EDP when a three (left-hand side) or a five (right-hand side) pole Butterworth electrical filter with $B_{e}=18 \mathrm{GHz}$ is considered at the $\mathrm{RX}$ side. An ideal $\mathrm{RX}$ is considered. Marks: MC simulation; lines: GA estimated from the results of MC simulation.

The PDF of the EDP has also been assessed for 67\% duty-cycle RZ-DQPSK signals for both types of electrical filters, leading to similar conclusions to those presented in this section.

\section{Gaussian approximation for equivalent differential phase}

The GA consists in approximating a given PDF by a Gaussian PDF. In order to do so, the mean and STD of the Gaussian PDF are set equal to the mean and STD of the PDF it is approximating. The mean and STD of the EDP are derived in this section as a function of the received DQPSK signal and PSD of optical noise at the RX input in order to obtain closedform expressions for the mean and STD of the EDP. Substituting eq. (2) in eq. (6) and setting $d=t-T_{M Z D I}$ to simplify the expressions, we get: 


$$
\begin{aligned}
\Delta \phi_{e}^{(I, Q)}(t)=\arg \{( & R_{1} / 2\left[\gamma\left(s(t) s^{*}(d)+s(t) n_{\|}^{*}(d)+n_{\|}(t) s^{*}(d)+n_{\|}(t) n_{\|}^{*}(d)+n_{\perp}(t) n_{\perp}^{*}(d)\right) e^{j \theta}\right] \\
+ & R_{1} / 4\left[\gamma^{2}\left(|s(d)|^{2}+2 \Re\left\{s(d) n_{\|}^{*}(d)\right\}+\left|n_{\|}(d)\right|^{2}+\left|n_{\perp}(d)\right|^{2}\right)\right. \\
& \left.+|s(t)|^{2}+2 \Re\left\{s(t) n_{\|}^{*}(t)\right\}+\left|n_{\|}(t)\right|^{2}+\left|n_{\perp}(t)\right|^{2}\right] \\
+ & R_{2} / 2\left[\gamma \left(s(t+\tau) s^{*}(d+\tau)+s(t+\tau) n_{\|}^{*}(d+\tau)+n_{\|}(t+\tau) s^{*}(d+\tau)\right.\right. \\
& \left.\left.+n_{\|}(t+\tau) n_{\|}^{*}(d+\tau)+n_{\perp}(t+\tau) n_{\perp}^{*}(d+\tau)\right) e^{j \theta}\right] \\
- & R_{2} / 4\left[\gamma^{2}\left(|s(d+\tau)|^{2}+2 \Re\left\{s(d+\tau) n_{\|}^{*}(d+\tau)\right\}+\left|n_{\|}(d+\tau)\right|^{2}+\left|n_{\perp}(d+\tau)\right|^{2}\right\}\right. \\
& \left.\left.\left.+|s(t+\tau)|^{2}+2 \Re\left\{s(t+\tau) n_{\|}^{*}(t+\tau)\right\}+\left|n_{\|}(t+\tau)\right|^{2}+\left|n_{\perp}(t+\tau)\right|^{2}\right]\right) * h_{e}(t)\right\}
\end{aligned}
$$

In order to obtain closed-form expressions for the mean and STD of the EDP, the dependence of the EDP on noise is linearized. This approximation should lead to only very small discrepancies in the mean and STD of the EDP as the EDP conditioned on the transmitted symbols is approximately Gaussian-distributed. The linearization of the EDP is performed expressing the argument of eq. (7) as an arctangent function. Thus, the several beat terms of eq. (7) are decomposed in their real and imaginary parts. The several beat terms can be written and defined as shown in eq. (14) and eq. (15) (Appendix 9.1). The time dependence of the DQPSK signal and noise is omitted in order to simplify the notation. By substituting the results shown in eqs. (14) and (15) in eq. (7) and by approximating the EDP by a first order Taylor series we get

$$
\begin{aligned}
\Delta \phi_{e}^{(I, Q)}(t)=\arctan (k)+\frac{k}{1+} k^{2}( & \gamma \frac{R_{1}}{2}\left(c_{2}\left[s n_{1, r}+s n_{2, r}+n n_{\|, r}+n n_{\perp, r}\right]\right. \\
& +c_{1}\left[s n_{1, i}+s n_{2, i}+n n_{\|, i}+n n_{\perp, i}\right) \\
+ & \gamma \frac{R_{2}}{2}\left(c_{2}\left[s n_{\tau, 1, r}+s n_{\tau, 2, r}+n n_{\tau, \|, r}+n n_{\tau, \perp, r}\right]\right. \\
& +c_{1}\left[s n_{\tau, 1, i}+s n_{\tau, 2, i}+n n_{\tau, \|, i}+n n_{\tau, \perp, i}\right) \\
& -\frac{R_{1}}{4 B}\left(2 \gamma^{2} s n_{d}+\gamma^{2} n n_{d, \|}+2 s n_{t}+n n_{t, \|}+\gamma^{2} n n_{d, \perp}+n n_{t, \perp}\right) \\
+ & \left.\frac{R_{2}}{4 B}\left(2 \gamma^{2} s n_{\tau, d}+\gamma^{2} n n_{\tau, d, \|}+2 s n_{\tau, t}+n n_{\tau, t, \|}+\gamma^{2} n n_{\tau, d, \perp}+n n_{\tau, t, \perp}\right)\right)
\end{aligned}
$$

where

$$
\begin{aligned}
A= & \gamma\left(\frac{R_{1}}{2}\left[s s_{i} \cos (\theta)+s s_{r} \sin (\theta)\right]+\frac{R_{2}}{2}\left[s s_{\tau, i} \cos (\theta)+s s_{\tau, r} \sin (\theta)\right]\right) \\
B= & \gamma\left(\frac{R_{1}}{2}\left[s s_{r} \cos (\theta)-s s_{i} \sin (\theta)\right]+\frac{R_{2}}{2}\left[s s_{\tau, r} \cos (\theta)-s s_{\tau, i} \sin (\theta)\right]\right) \\
& +\frac{R_{1}}{4}\left[\gamma^{2} s s_{d}+s s_{t}\right]-\frac{R_{2}}{4}\left[\gamma^{2} s s_{\tau, d}+s s_{\tau, t}\right] ; \\
k= & A / B ; \quad c_{1}=\cos (\theta) / A+\sin (\theta) / B ; \quad c_{2}=\sin (\theta) / A-\cos (\theta) / B ;
\end{aligned}
$$


From eq. (8), the mean of the EDP is

$$
\begin{aligned}
\mu(t)=\arctan (k)+\frac{k}{1+k^{2}} & \left(\gamma \frac{R_{1}}{2}\left[c_{2}\left(\mathrm{E}\left\{n n_{\|, r}\right\}+\mathrm{E}\left\{n n_{\perp, r}\right\}\right)+c_{1}\left(\mathrm{E}\left\{n n_{\|, i}\right\}+\mathrm{E}\left\{n n_{\perp, i}\right\}\right]\right)\right. \\
& +\gamma \frac{R_{2}}{2}\left[c_{2}\left(\mathrm{E}\left\{n n_{\tau, \|, r}\right\}+\mathrm{E}\left\{n n_{\tau, \perp, r}\right\}\right)+c_{1}\left(\mathrm{E}\left\{n n_{\tau, \|, i}\right\}+\mathrm{E}\left\{n n_{\tau, \perp, i}\right\}\right]\right] \\
& -\frac{R_{1}}{4 B}\left(\gamma^{2} \mathrm{E}\left\{n n_{d, \|}\right\}+\mathrm{E}\left\{n n_{t, \|}\right\}+\gamma^{2} \mathrm{E}\left\{n n_{d, \perp}\right\}+\mathrm{E}\left\{n n_{t, \perp}\right\}\right) \\
& \left.+\frac{R_{2}}{4 B}\left[\gamma^{2} \mathrm{E}\left\{n n_{\tau, d, \|}\right\}+\mathrm{E}\left\{n n_{\tau, t, \|}\right\}+\gamma^{2} \mathrm{E}\left\{n n_{\tau, d, \perp}\right\}+\mathrm{E}\left\{n n_{\tau, t, \perp}\right\}\right]\right)
\end{aligned}
$$

Assuming uncorrelated noise over both polarization directions, i.e., $\mathrm{E}\left(n n_{\|, x} n n_{\perp, y}\right)=\mathrm{E}\left(n n_{\|, x}\right) \mathrm{E}\left(n n_{\perp, y}\right)$, where $x$ and $y$ represent the real or imaginary part of noisenoise beat terms and, as odd order moments of Gaussian processes with zero mean are null, the variance of the EDP is given by:

$$
\sigma^{2}(t)=\left(\frac{k}{1+k^{2}}\right)^{2}\left(\sum_{l=1}^{8}\left[\sigma_{N, s-A S E, l}^{2}+\sigma_{N, A S E-A S E, l}^{2}\right]\right)
$$

where $\sigma_{N, S-A S E, l}^{2}$ and $\sigma_{N, A S E-A S E, l}^{2}$ are the contributions to the signal and noise-noise beat variance, respectively, presented in Appendices 9.2 and 9.3. The variance of the EDP (eq. (11)) is given by a lenghty expression. However, the evaluation of the several terms of eq.

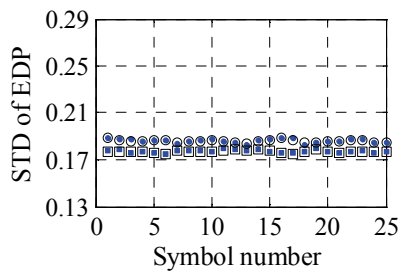

a) Ideal RX

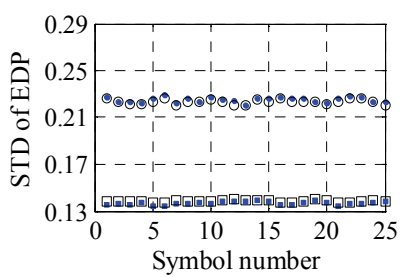

d) $R_{1}=0.5 \mathrm{~A} / \mathrm{W}, R_{2}=1 \mathrm{~A} / \mathrm{W}$

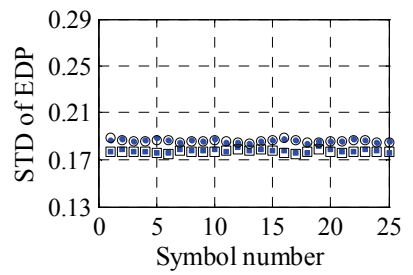

b) $\Delta f / B_{R}=2 \%$

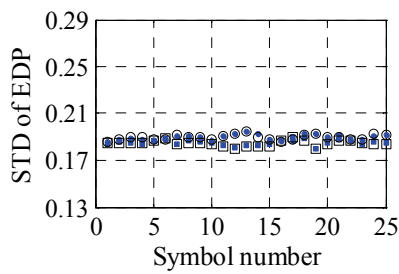

e) $\delta T_{M Z D I} / T_{S}=20 \%$

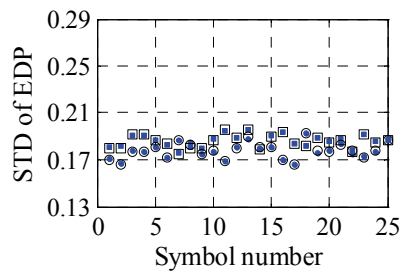

c) $\Delta \tau / T_{S}=40 \%$

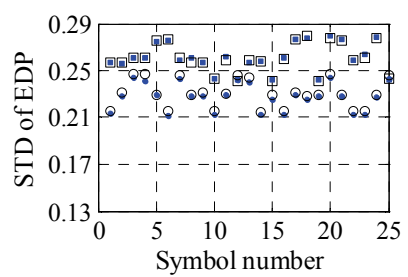

f) $\Delta v=20 \mathrm{GHz}$

Fig. 8. Standard deviation of the EDP. Only the STD of the EDP of some symbols transmitted with two of the four nominal means (circles: $\pi / 4$; squares: $-3 \pi / 4$ ) is shown in order to make the figures clearer. Filled symbols: estimates from MC simulation results, obtained considering 15000 noise realizations; empty symbols: estimates from the GA (eq. (11)). 
(11) is quite simple which makes the evaluation of the variance of the EDP of quite reduced complexity. Furthermore, if no RX imperfections are considered, eq. (11) is quite simplified, leading to the result shown in (Costa \& Cartaxo, 2009). The derivation of the mean and variance of EDP as a function of the received DQPSK signal and PSD of optical noise after optical filtering is shown in (Costa \& Cartaxo, 2009b)

Fig. 8 shows the STD of the EDP estimated using the results from MC simulation and the GA (eq. (11)). Analysis of Fig. 8 shows that the estimates of the STD of the EDP obtained using eq. (11) are quite accurate in presence of the majority of RX imperfections. The accuracy of the estimates for the mean of the EDP, estimated using eq. (10), has also been assessed showing that the mean of the EDP is always quite well estimated by eq. (10). The quite good accuracy achieved in the estimation of the mean and STD of the EDP using eqs. (10) and (11) shows that the linearization of the EDP leads only to very small discrepancies on the evaluation of the mean and STD of the EDP and that the impact of noise on the mean and STD of the EDP is correctly estimated.

\section{Bit error probability computation by semi-analytical simulation method}

A SASM for performance evaluation of DQPSK systems is proposed in this section. The DQPSK signal at the RX input is evaluated by simulation. This permits evaluating the impact of the transmission path, e.g. the nonlinear fiber transmission, the optical add-drop multiplexer concatenation filtering, on the waveform of the DQPSK signal. A quaternary deBruijn sequence with total length $N_{S}$ is used in the simulation. DeBruijn sequences include all possible symbol sequences with a given length using the lower number of symbols (Jeruchim et al., 2000). This characteristic is important since it assures that all possible cases of inter-symbol interference (ISI) for a given sequence length occur. On the other hand, as the EDP is approximately Gaussian-distributed when the optical noise is modelled as AWGN at the RX input, the impact of noise on the DQPSK system performance is assessed analytically.

As the precoding performed in the TX allows direct mapping of the bit sequence from the TX input to the RX output, the overall BEP is given by $B E P=\left(B E P^{(I)}+B E P^{(Q)}\right) / 2$, where $B E P^{(I, Q)}$ is the BEP of each component of the DQPSK signal. In order to take accurately into account the impact of ISI on the DQPSK system performance, separate Gaussian distributions with different means and STDs are associated with each one of the transmitted bits. This approach has already proved to be accurate to estimate the ISI impact on OOK modulation (Rebola \& Cartaxo, 2001). The BEP of each component of the DQPSK signal can be seen as the mean of four BEPs associated with the four nominal means for the PDF of the EDP. Thus, defining $F$ as the EDP threshold level, with $F \geq 0$, the BEP of the I and Q components of the DQPSK signal is given by

$$
B E P^{(I, Q)}=\frac{1}{2 N_{S}}\left(\sum_{\substack{n=1 \\ a_{n}= \pm \pi / 4}}^{N_{s}} \operatorname{erfc}\left[\frac{F-\left|\mu_{a_{n}, n}\right|}{\sqrt{2} \sigma_{a_{n}, n}}\right]+\sum_{\substack{n=1 \\ a_{n}= \pm 3 \pi / 4}}^{N_{s}} \operatorname{erfc}\left[\frac{-F+\left|\mu_{a_{n}, n}\right|}{\sqrt{2} \sigma_{a_{n}, n}}\right]\right)
$$

where $\operatorname{erfc}(x)$ is the complementary error function and $\mu_{a_{n}, n}$ and $\sigma_{a_{n}, n}$ are the mean and STD of the EDP at the sampling time for the $n$-th received symbol with nominal mean $a_{n}$. 
$\mu_{a_{n}, n}$ and $\sigma_{a_{n}, n}$ are obtained from eq. (10) and eq. (11), respectively, by evaluating these expressions at the sampling time and by associating each sampling time with each transmitted symbol. The optimal threshold level of the EDP, $F_{\text {opt }}$, is assessed by setting to zero the derivative of eq. (12) with respect to $F$, leading to the transcendental equation

$$
\sum_{\substack{n=1 \\ a_{n}= \pm \pi / 4}}^{N_{s}} \frac{1}{\sigma_{a_{n}, n}} \exp \left(-\frac{1}{2}\left[\frac{F_{o p t}-\left|\mu_{a_{n}, n}\right|}{\sigma_{a_{n}, n}}\right]^{2}\right)=\sum_{\substack{n=1 \\ a_{n}= \pm 3 \pi / 4}}^{N_{s}} \frac{1}{\sigma_{a_{n}, n}} \exp \left(-\frac{1}{2}\left[\frac{-F_{o p t}+\left|\mu_{a_{n}, n}\right|}{\sigma_{a_{n}, n}}\right]^{2}\right)
$$

that can be numerically solved using the Newton-Raphson method.

\section{Accuracy of the SASM based on the GA for the EDP}

In this section, the accuracy of the SASM for DQPSK system performance evaluation based on the GA for the EDP is assessed. This analysis is performed comparing the results obtained using eq. (12) with those obtained using MC simulation. A BEP $=10^{-4}$ is set as the target BEP mainly because $\mathrm{MC}$ simulation is much time consuming for lower BEP and the use of forward error correction (FEC), such as Reed-Solomon codes, allows to achieve much lower BEP at the expense of only a slight increase on the bit rate. The accuracy of the SASM is firstly assessed in presence of RX imperfections. Then, the accuracy of the SASM is assessed considering nonlinear fiber transmission. The bit error ratio estimates obtained using MC simulation are only accepted after at least 100 errors occurring in each component of the DQPSK signal. The threshold level is optimized and the time instant leading to higher eye-opening in the absence of noise is chosen as sampling time. The TX and RX parameters are the same as the ones considered in section 3, unless otherwise stated.

\subsection{Accuracy of the SASM in presence of RX imperfections}

When the ideal RX is considered, the MC simulation estimates that an OSNR of about $14 \mathrm{~dB}$ is required to achieve $B E P=10^{-4}$. The SASM estimates a required OSNR of only about $13.8 \mathrm{~dB}$. This small difference is attributed mainly to the difference between the GA for the PDF of the EDP and its actual PDF. This conclusion results from having very good agreement between the estimates of the mean and STD of the EDP obtained using eq. (10) and eq. (11) with the corresponding ones obtained using MC simulation. Indeed, the SASM leads to the correct required OSNR $(14 \mathrm{~dB})$ by increasing the STD of the EDP, calculated using eq. (11), by only about $2.5 \%$.

Fig. 9 shows the impact of several different RX imperfections on the OSNR penalty at $B E P=10^{-4}$. The considered $\mathrm{RX}$ imperfections cover all expected values for each imperfection. The impact of the RX imperfections on the DQPSK system performance has been assessed by MC simulation and by SASM in order to assess the accuracy of the SASM. The analysis of Fig. 9 shows that the SASM is quite accurate in presence of the majority of the typical RX imperfections leading usually to a discrepancy on the OSNR penalty not exceeding $0.2 \mathrm{~dB}$. Among the cases analysed in Fig. 9, the higher discrepancies occur for high timemisalignment of signals at the balanced detector input $(|\Delta \tau / T|>30 \%)$ and for high frequency detuning of the optical filters $(|\Delta v|>15 \mathrm{GHz})$. Indeed, the SASM leads to an underestimation of the OSNR penalty in both cases that may attain about $0.5 \mathrm{~dB}$. 


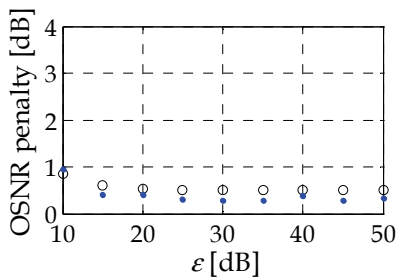

a) MZDI extinction ratio with $k=0.3$

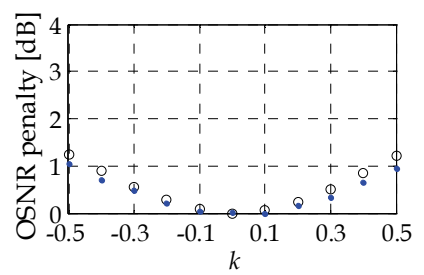

d) Amplitude-imbalance of balanced detector

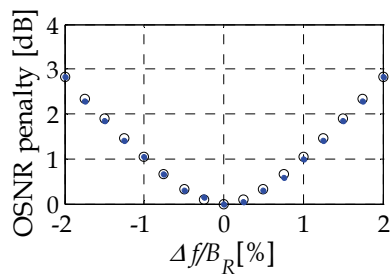

b) MZDI detuning

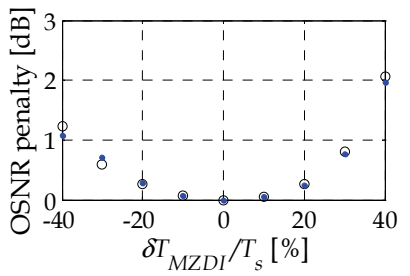

e) MZDI delay error

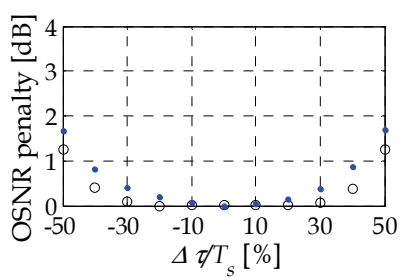

c) Time-misalignment of signals at balanced detector input

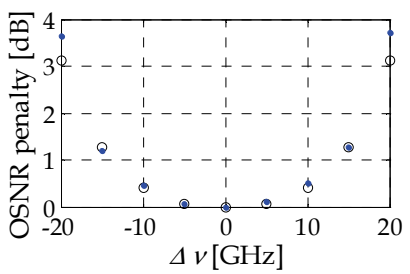

f) Optical filter detuning

Fig. 9. OSNR penalty at $B E P=10^{-4}$ as a function of several different $\mathrm{RX}$ imperfections. Filled circles: MC simulation; empty circles: SASM.

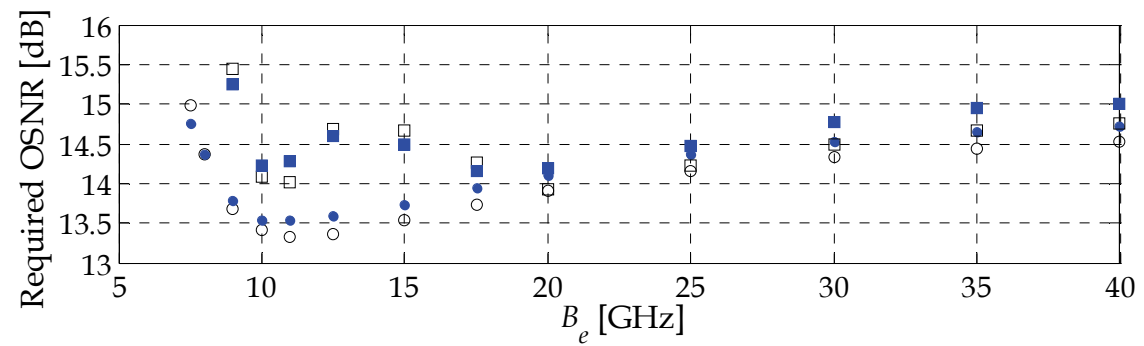

Fig. 10. Required OSNR at $B E P=10^{-4}$ as a function of the electrical filter type and bandwidth, considering an ideal RX. Empty marks: SASM; filled marks: MC simulation. Circles: five-pole Bessel electrical filter; squares: five-pole Butterworth electrical filter.

Fig. 10 illustrates the accuracy of the SASM when different bandwidths and types of electrical filter are considered. Fig. 10 shows that the required OSNR is quite well estimated independently of the type and bandwidth of the electrical filter. Indeed, the discrepancy of the required OSNR does not usually exceed $0.2 \mathrm{~dB}$. This small discrepancy is mainly attributed to the difference between the GA for the PDF of the EDP and its actual PDF. Fig. 10 shows also that the behavior of the required OSNR as a function of the electrical filter bandwidth depends on the electrical filter type. The different behaviors illustrated in Fig. 10 for filter bandwidths around $12 \mathrm{GHz}$ can be explained by observing the eye-opening. Indeed, we find that the eye-opening is more reduced for $B_{e}$ around $12.5 \mathrm{GHz}$ than for $B_{e}$ around $11 \mathrm{GHz}$ when the Butterworth electrical filter is used, which does not occur in case of the Bessel electrical filter. 


\subsection{Accuracy of the SASM in presence of nonlinear fiber transmission}

To reach long-haul cost-efficient transmission, as required in core networks, the fiber spans should be quite long to reduce the number of required optical amplifiers. The power level at the input of each span should also be as high as possible to achieve high OSNR. On the other hand, when high power levels are used, the fiber nonlinearity imposes a severe power penalty. Thus, a compromise between the optical power level and the power penalty imposed by the fiber nonlinearity has to be accomplished. Standard single-mode fiber (SSMF) is the transmission fiber type more commonly used in these networks. Despite its many advantages, it introduces high distortion in the transmitted signal due to its high dispersion. Thus, the use of dispersion compensation along the transmission path is required.

In an ideal single-mode optical fiber, the two orthogonal states of polarization are degenerated, i. e. they propagate with identical propagation constants (Iannone et al., 1998). Thus, the input light-polarization would remain constant over the whole propagation length. In reality, optical fibers may have a slightly elliptical core which leads to birefringence, i. e. the propagation constants of the two orthogonal states of polarization differ slightly. External perturbations such as stress, bending and torsion lead also to birefringence (Hanik, 2002). Thus, the impact of fiber birefringence, group velocity dispersion (GVD) and self-phase modulation (SPM) are considered to assess the accuracy of the SASM in presence of nonlinear fiber transmission.

The MC simulation is performed by solving the coupled nonlinear Schrödinger propagation equation, also known as the vector version of the nonlinear Schrödinger propagation equation, instead of the scalar version of the nonlinear Schrödinger propagation equation, in order to take into account the impact of fiber birefringence. However, the solution of the coupled nonlinear Schrödinger propagation equation is much more complex than the one of the scalar version (Iannone et al., 1998). Nevertheless, the split-step Fourier method, which is usually used to solve the scalar version of the nonlinear Schrödinger propagation equation, can be applied to its vector version when the so-called high-birefringence condition (Iannone et al., 1998) is verified. In this case, the exponential term in the vector version of the nonlinear Schrödinger propagation equation that depends on the birefringence fluctuates rapidly and its effect tends to average out (Iannone et al., 1998). This approximation is usually verified in single mode optical fibers and has been commonly used in the literature where negligible loss of accuracy is usually achieved (Marcuse et al., 1997). Furthermore, by choosing an adequate integration step, the coupling between the polarization modes can be neglected when solving the propagation within a single step. After each step, the eigenpolarizations are randomly rotated and a random phase shift is added. A more detailed explanation of how the simulation of fiber nonlinear transmission is performed can be found in (Iannone et al., 1998). In our MC simulation, the birefringence is assumed constant over successive integration steps of 100 meters. The eigenpolarizations are uniformly distributed over the birefringence axes and the phase shift, which corresponds to $2 \pi$ over the beat length, has a Rician distribution with mean value $0.1 \cdot 2 \pi \mathrm{m}^{-1}$ and variance $0.01 \cdot 2 \pi \mathrm{m}^{-1}$ (Carena et al., 1998).

The DQPSK system performance evaluation by the SASM requires assessing the DQPSK noiseless waveform and PSD of optical noise at RX input after nonlinear fiber transmission. The noiseless waveform of the DQPSK signal is assessed by performing noiseless 
transmission of the DQPSK signal using the scalar version of the nonlinear Schrödinger equation, but with the fiber nonlinearity coefficient reduced by a $8 / 9$ factor. Indeed, the scalar version of the nonlinear Schrödinger propagation equation leads to similar results to those of its vector version when it is solved with the fiber nonlinearity coefficient set to 8/9 of its real value (Carena et. al., 1998), (Hanik, 2002). The PSD of optical noise depends on the polarization direction. Indeed, the AWGN approximation for optical noise at the RX input over the same polarization direction as the DQPSK signal may be quite inaccurate when nonlinear fiber transmission is considered. Indeed, when a strong signal (the DQPSK signal) propagates along a transmission fiber, it creates a spectral region around itself where a small signal (the optical amplifier's ASE noise) experiences gain. This phenomenon is known as parametric gain (Carena et al., 1998). Furthermore, the nonlinear phase noise due to the amplitude-to-phase noise conversion effect arising from the interaction of the optical amplifier's ASE noise and the nonlinear Kerr effect must also be taken into account. The evaluation of the parametric gain and nonlinear phase noise can be performed considering the nonlinear fiber transmission of only one polarization direction but with the fiber nonlinearity coefficient reduced by the $8 / 9$ factor. This approximation allows evaluating the PSD of optical noise after nonlinear fiber transmission over the DQPSK signal polarization direction using the method proposed in (Demir, 2007). The method proposed in (Demir, 2007) evaluates the PSD of optical noise in a quite time efficient manner by deriving a linear partial-differential equation for the noise perturbation. In order to do so, the nonlinear Schrödinger equation is linearized around a continuous-wave signal. The AWGN approximation for optical noise at $\mathrm{RX}$ input over the perpendicular polarization direction is still quite accurate (Carena et al., 1998). Thus, the PSD of optical noise over the perpendicular polarization direction is obtained by adding the individual ASE noise contributions of each optical amplifier, each affected by the total gain from the optical amplifier till the RX.

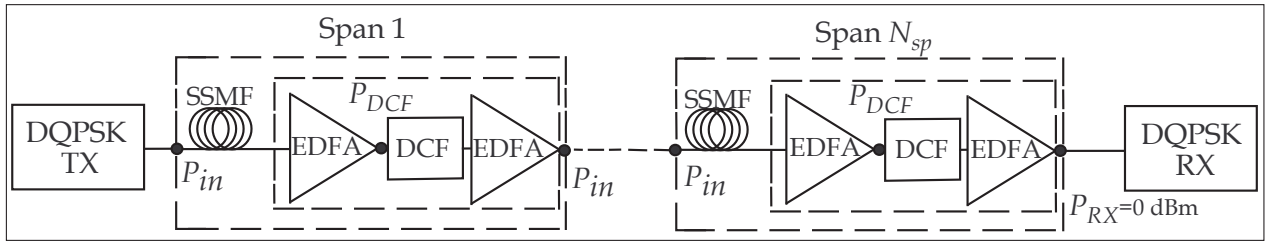

Fig. 11. Scheme of the DQPSK transmission system.

Fig. 11 shows the schematic configuration of the DQPSK transmission system. The total link is composed by $N_{s p}$ spans, with $N_{s p}=20$. Each span is composed by $100 \mathrm{~km}$ of SSMF followed by a double-stage erbium-doped fiber amplifier (EDFA). Dispersion compensating fibers (DCFs) are used for total compensation of the dispersion accumulated in the SSMF of each span. To assure that all DCFs operate nearly in linear regime, the power level denoted by $P_{D C F}$ is imposed at the DCFs input. The average power level at the input of each SSMF is denoted by $P_{i n}$. The total gain of both EDFAs' stages compensates for the power loss in each span, except in the last span. In this case, the second stage EDFA is used to impose a power level of $0 \mathrm{dBm}$ at the RX input. The SSMF has an attenuation parameter of $0.21 \mathrm{~dB} / \mathrm{km}$, a dispersion parameter of $17 \mathrm{ps} / \mathrm{nm} / \mathrm{km}$, an effective core area of $80 \mu \mathrm{m}^{2}$ and a nonlinear index-coefficient of $0.025 \mathrm{~nm}^{2} / \mathrm{W}$. The EDFA's noise figure is $7 \mathrm{~dB}$. The dispersion parameter of the DCF is $-100 \mathrm{ps} / \mathrm{nm} / \mathrm{km}$ and its attenuation parameter depends on the 
transmission scenario that is being considered. Indeed, in order to keep the BEP high enough to perform MC simulation in a reasonable amount of time and, as 33\% duty-cycle RZ-DQPSK pulses show better performance than NRZ-DQPSK pulses, the attenuation parameter of DCF is $0.5 \mathrm{~dB} / \mathrm{km}$ when NRZ-DQPSK pulses are considered and $0.6 \mathrm{~dB} / \mathrm{km}$ when $33 \%$ duty-cycle RZ-DQPSK pulses are considered. Furthermore, $P_{D C F}=-8 \mathrm{dBm}$ when NRZ-DQPSK pulses are considered and $P_{D C F}=-12 \mathrm{dBm}$ when $33 \%$ duty-cycle RZDQPSK pulses are considered.
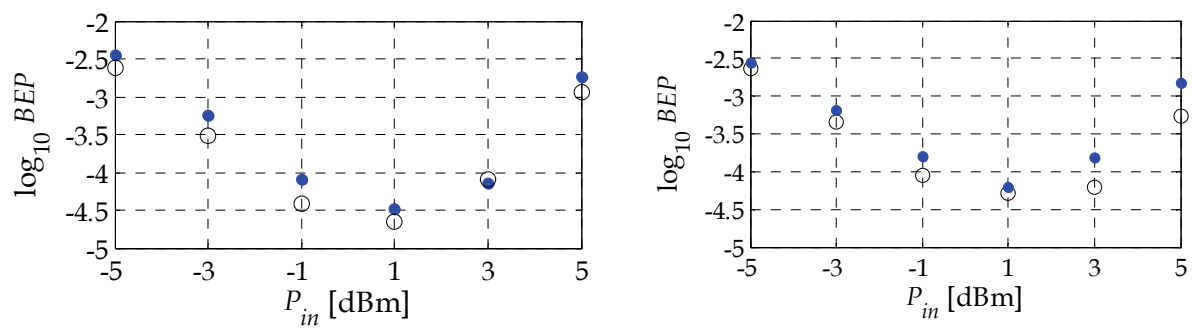

Fig. 12. Performance of NRZ-DQPSK (left) and 33\% duty-cycle RZ-DQPSK (right) for the transmission system of Fig. 11. Filled circles: MC simulation; empty circles: SASM.

Fig. 12 shows the performance of DQPSK modulation in presence of nonlinear fiber transmission. When NRZ-DQPSK signals are considered (figure on the left), a good accuracy is achieved both when ASE noise is the main transmission impairment (lower power levels) and when the transmission is mainly limited by fiber nonlinearity (higher power levels). This result leads to the conclusion that methods for DQPSK system performance evaluation based on the GA for the EDP lead to quite good accuracy even in presence of nonlinear fiber transmission when NRZ-DQPSK signals are considered. The analysis of the figure on the right-hand side, where the transmission of a 33\% duty-cycle RZ-DQPSK signal is considered, shows that the SASM estimates the performance of the DQPSK system quite accurately when ASE noise is the main impairment. However, the accuracy of the SASM tends to decrease with the increase of the impact of the fiber nonlinearity. This loss of accuracy is not a consequence of the loss of accuracy of the GA for the EDP. Indeed, quite good accuracy is achieved when the mean and STD of the EDP are estimated from the results of MC simulation. The decrease of accuracy of the SASM is a consequence of the inaccuracy in the evaluation of the PSD of optical noise. Indeed, the linearization of the nonlinear Schrödinger equation around a continuous-wave signal does not provide an acceptable description of noise statistics when 33\% duty-cycle RZ-DQPSK signals are transmitted and the impact of fiber nonlinearity is important.

Computation time gains of about 15000 times have been achieved by the SASM when compared with MC simulation for $B E P=10^{-4}$.

\section{Conclusion and work in progress}

The performance evaluation of simulated optical DQPSK modulation has been analysed. The EDP of DQPSK signals is approximately Gaussian-distributed. Thus, a SASM for DQPSK systems performance evaluation based on the GA has been proposed. The SASM relies on the use of the closed-form expressions derived for the mean and STD of the EDP 
for assessing the performance of the DQPSK system in a time-efficient manner. Quite good agreement between MC simulation and the results of the SASM is usually achieved, even in presence of RX imperfections and nonlinear fiber transmission. Indeed, although the SASM leads usually to an underestimation of the required OSNR of about $0.2 \mathrm{~dB}$, the discrepancy of the OSNR penalty at $B E P=10^{-4}$ is usually below $0.2 \mathrm{~dB}$ for the majority of the typical $\mathrm{RX}$ imperfections.

Several subjects on the performance evaluation of DQPSK signals using the GA for the EDP are still to be addressed. Indeed, the evaluation of the PSD of optical noise at RX input, after nonlinear transmission, admitting a modulated signal and the accuracy improvement of the SASM achieved by using the more accurate description for the PSD of optical noise is still to be performed. The validation of the SASM for BEP around $10^{-12}$ and the proposal of a scheme for evaluating the EDP experimentally are also still to be addressed.

\section{Acknowledgments}

This work was supported in part by Fundação para a Ciência e a Tecnologia from Portugal under Ph.D. contract SFRH/BD/42287/2007.

\section{Appendix}

\subsection{List of beat terms at decision circuit Input}

The current and the EDP at the decision circuit input are given as a function of the following beat terms:

$$
\begin{aligned}
& \left(s(t) s^{*}(d)\right) * h_{e}(t)=\left(s_{r}(t) s_{r}(d)+s_{i}(t) s_{i}(d)+j\left[s_{i}(t) s_{r}(d)-s_{r}(t) s_{i}(d)\right]\right) * h_{e}(t) \equiv s s_{r}+j s s_{i} \\
& \left(s(t) n_{\|}^{*}(d)\right) * h_{e}(t)=\left(s_{r}(t) n_{\|, r}(d)+s_{i}(t) n_{\|, i}(d)+j\left[s_{i}(t) n_{\|, r}(d)-s_{r}(t) n_{\|, i}(d)\right]\right) * h_{e}(t) \equiv s n_{1, r}+j s n_{1, i} \\
& \left(n_{\|}(t) s^{*}(d)\right) * h_{e}(t)=\left(n_{\|, r}(t) s_{r}(d)+n_{\|, i}(t) s_{i}(d)+j\left[n_{\|, i}(t) s_{r}(d)-n_{\|, r}(t) s_{i}(d)\right]\right) * h_{e}(t) \equiv s n_{2, r}+j s n_{2, i} \\
& \left(n_{\|}(t) n_{\|}^{*}(d)\right) * h_{e}(t)=\left(n_{\|, r}(t) n_{\|, r}(d)+n_{\|, i}(t) n_{\|, i}(d)+j\left[n_{\|, i}(t) n_{\|, r}(d)-n_{\|, r}(t) n_{\|, i}(d)\right]\right) * h_{e}(t) \\
& \quad \equiv n n_{\|, r}+j n n_{\|, i} \\
& \left(n_{\perp}(t) n_{\perp}^{*}(d)\right) * h_{e}(t)=\left(n_{\perp, r}(t) n_{\perp, r}(d)+n_{\perp, i}(t) n_{\perp, i}(d)+j\left[n_{\perp, i}(t) n_{\perp, r}(d)-n_{\perp, r}(t) n_{\perp, i}(d)\right]\right) * h_{e}(t) \\
& \quad \equiv n n_{\perp, r}+j n n_{\perp, i} \\
& |s(d)|^{2} * h_{e}(t)=\left(s_{r}^{2}(d)+s_{i}^{2}(d)\right) * h_{e}(t) \equiv s s_{d} \\
& \Re\left\{s(d) n_{\|}^{*}(d)\right\} * h_{e}(t)=\left(s_{r}(d) n_{\|, r}(d)+s_{i}(d) n_{\|, i}(d)\right) * h_{e}(t) \equiv s n_{d} \\
& |s(t)|^{2} * h_{e}(t)=\left(s_{r}^{2}(t)+s_{i}^{2}(t)\right) * h_{e}(t) \equiv s s_{t} \\
& \left|n_{\|}(d)\right|^{2} * h_{e}(t)=\left(n_{\|, r}^{2}(d)+n_{\|, i}^{2}(d)\right) * h_{e}(t) \equiv n n_{d, \|} \\
& \Re\left\{s(t) n_{\|}^{*}(t)\right\} * h_{e}(t)=\left(s_{r}(t) n_{\|, r}(t)+s_{i}(t) n_{\|, i}(t)\right) * h_{e}(t) \equiv s n_{t} \\
& \left|n_{\|}(t)\right|^{2} * h_{e}(t)=\left(n_{\|, r}^{2}(t)+n_{\|, i}^{2}(t)\right) * h_{e}(t) \equiv n n_{t, \|} \\
& \left|n_{\perp}(d)\right|^{2} * h_{e}(t)=\left(n_{\perp, r}^{2}(d)+n_{\perp, i}^{2}(d)\right) * h_{e}(t) \equiv n n_{d, \perp} \\
& \left|n_{\perp}(t)\right|^{2} * h_{e}(t)=\left(n_{\perp, r}^{2}(t)+n_{\perp, i}^{2}(t)\right) * h_{e}(t) \equiv n n_{t, \perp}
\end{aligned}
$$


and, similarly:

$$
\begin{aligned}
& \left(s(t+\tau) s^{*}(d+\tau)\right) * h_{e}(t) \equiv s s_{\tau, r}+j s s_{\tau, i} ; \quad\left(s(t+\tau) n_{\|}^{*}(d+\tau)\right) * h_{e}(t) \equiv s n_{\tau, 1, r}+j s n_{\tau, 1, i} \\
& \left(n_{\|}(t+\tau) s^{*}(d+\tau)\right) * h_{e}(t) \equiv s n_{\tau, 2, r}+j s n_{\tau, 2, i} ; \quad\left(n_{\|}(t+\tau) n_{\|}^{*}(d+\tau)\right) * h_{e}(t) \equiv n n_{\tau, \|, r}+j n n_{\tau, \|, i} \\
& \left(n_{\perp}(t+\tau) n_{\perp}^{*}(d+\tau)\right) * h_{e}(t) \equiv n n_{\tau, \perp, r}+j n n_{\tau, \perp, i} ; \quad|s(d+\tau)|^{2} * h_{e}(t) \equiv s s_{\tau, d} \\
& \Re\left\{s(d+\tau) n_{\|}^{*}(d+\tau)\right\} * h_{e}(t) \equiv s n_{\tau, d} ; \quad|s(t+\tau)|^{2} * h_{e}(t) \equiv s s_{\tau, t} \\
& \left|n_{\|}(d+\tau)\right|^{2} * h_{e}(t) \equiv n n_{\tau, d, \|} ; \quad \Re\left\{s(t+\tau) n_{\|}^{*}(t+\tau)\right\} * h_{e}(t) \equiv s n_{\tau, t} \\
& \left|n_{\|}(t+\tau)\right|^{2} * h_{e}(t) \equiv n n_{\tau, t, \|} ; \quad\left|n_{\perp}(d+\tau)\right|^{2} * h_{e}(t) \equiv n n_{\tau, d, \perp} \\
& \left|n_{\perp}(t+\tau)\right|^{2} * h_{e}(t) \equiv n n_{\tau, t, \perp}
\end{aligned}
$$

\subsection{Contributions to the signal-noise beat variance}

The variance of the signal-noise beat may be separated in several contributions. To illustrate the impact of RX imperfections, the contributions to the signal-noise beat variance of ideal RX, shown in (Costa \& Cartaxo, 2009b), are used as reference. Thus, we find that the $c_{2}^{2}\left(\mathrm{E}\left\{\left(s n_{1, r}\right)^{2}\right\}+2 \mathrm{E}\left\{s n_{1, r} s n_{2, r}\right\}+\mathrm{E}\left\{\left(s n_{2, r}\right)^{2}\right\}\right)$ contribution to the signal-noise beat variance results from

$$
\begin{aligned}
\sigma_{N, s-A S E, 1}^{2}= & \gamma^{2} \frac{R_{1}^{2}}{4} c_{2}^{2}\left(\mathrm{E}\left\{\left(s n_{1, r}\right)^{2}\right\}+2 \mathrm{E}\left\{s n_{1, r} s n_{2, r}\right\}+\mathrm{E}\left\{\left(s n_{2, r}\right)^{2}\right\}\right) \\
& +\gamma^{2} \frac{R_{2}^{2}}{4} c_{2}^{2}\left(\mathrm{E}\left\{\left(s n_{\tau, 1, r}\right)^{2}\right\}+2 \mathrm{E}\left\{s n_{\tau, 1, r} s n_{\tau, 2, r}\right\}+\mathrm{E}\left\{\left(s n_{\tau, 2, r}\right)^{2}\right\}\right) \\
& +\gamma^{2} \frac{R_{1} R_{2}}{2} c_{2}^{2}\left(\mathrm{E}\left\{s n_{1, r} s n_{\tau, 1, r}\right\}+\mathrm{E}\left\{s n_{1, r} s n_{\tau, 2, r}\right\}+\mathrm{E}\left\{s n_{2, r} s n_{\tau, 1, r}\right\}+\mathrm{E}\left\{s n_{2, r} s n_{\tau, 2, r}\right\}\right)
\end{aligned}
$$

by considering an ideal RX. Similarly, the $c_{1}^{2}\left(E\left\{\left(s n_{1, i}\right)^{2}\right\}+2 E\left\{s n_{1, i} s n_{2, i}\right\}+E\left\{\left(s n_{2, i}\right)^{2}\right\}\right)$ and $2 c_{1} c_{2}\left(\mathrm{E}\left\{s n_{1, r} s n_{1, i}\right\}+\mathrm{E}\left\{s n_{2, r} s n_{1, i}\right\}+\mathrm{E}\left\{s n_{1, r} s n_{2, i}\right\}+\mathrm{E}\left\{s n_{2, r} s n_{2, i}\right\}\right)$ contributions to the signal-noise beat variance result from

$$
\begin{aligned}
\sigma_{N, s-A S E, 2}^{2}= & \gamma^{2} \frac{R_{1}^{2}}{4} c_{1}^{2}\left(\mathrm{E}\left\{\left(s n_{1, i}\right)^{2}\right\}+2 \mathrm{E}\left\{s n_{1, i} s n_{2, i}\right\}+\mathrm{E}\left\{\left(s n_{2, i}\right)^{2}\right\}\right) \\
& +\gamma^{2} \frac{R_{2}^{2}}{4} c_{1}^{2}\left(\mathrm{E}\left\{\left(s n_{\tau, 1, i}\right)^{2}\right\}+2 \mathrm{E}\left\{s n_{\tau, 1, i} s n_{\tau, 2, i}\right\}+\mathrm{E}\left\{\left(s n_{\tau, 2, i}\right)^{2}\right\}\right) \\
& +\gamma^{2} \frac{R_{1} R_{2}}{2} c_{1}^{2}\left(\mathrm{E}\left\{s n_{1, i} s n_{\tau, 1, i}\right\}+\mathrm{E}\left\{s n_{1, i} s n_{\tau, 2, i}\right\}+\mathrm{E}\left\{s n_{2, i} s n_{\tau, 1, i}\right\}+\mathrm{E}\left\{s n_{2, i} s n_{\tau, 2, i}\right\}\right)
\end{aligned}
$$

and 


$$
\begin{aligned}
\sigma_{N, s-A S E, 3}^{2}= & \gamma^{2} \frac{R_{1}^{2}}{2} c_{1} c_{2}\left(\mathrm{E}\left\{s n_{1, r} s n_{1, i}\right\}+\mathrm{E}\left\{s n_{2, r} s n_{1, i}\right\}+\mathrm{E}\left\{s n_{1, r} s n_{2, i}\right\}+\mathrm{E}\left\{s n_{2, r} s n_{2, i}\right\}\right) \\
& +\gamma^{2} \frac{R_{1} R_{2}}{2} c_{1} c_{2}\left(\mathrm{E}\left\{s n_{\tau, 1, r} s n_{1, i}\right\}+\mathrm{E}\left\{s n_{\tau, 2, r} s n_{1, i}\right\}+\mathrm{E}\left\{s n_{\tau, 1, r} s n_{2, i}\right\}+\mathrm{E}\left\{s n_{\tau, 2, r} s n_{2, i}\right\}\right) \\
& +\gamma^{2} \frac{R_{1} R_{2}}{2} c_{1} c_{2}\left(\mathrm{E}\left\{s n_{1, r} s n_{\tau, 1, i}\right\}+\mathrm{E}\left\{s n_{1, r} s n_{\tau, 2, i}\right\}+\mathrm{E}\left\{s n_{2, r} s n_{\tau, 1, i}\right\}+\mathrm{E}\left\{s n_{2, r} s n_{\tau, 2, i}\right\}\right) \\
& +\gamma^{2} \frac{R_{2}^{2}}{2} c_{1} c_{2}\left(\mathrm{E}\left\{s n_{\tau, 1, r} s n_{\tau, 1, i}\right\}+\mathrm{E}\left\{s n_{\tau, 2, r} s n_{\tau, 1, i}\right\}+\mathrm{E}\left\{s n_{\tau, 1, r} s n_{\tau, 2, i}\right\}+\mathrm{E}\left\{s n_{\tau, 2, r} s n_{\tau, 2, i}\right\}\right)
\end{aligned}
$$

respectively, when the ideal $\mathrm{RX}$ is considered. Other contributions to the signal-noise beat variance arise from the imperfections of the $R X$ and are cancelled when the ideal $R X$ is considered. These contributions are:

$$
\begin{aligned}
\sigma_{N, s-A S E, 4}^{2}= & \gamma \frac{R_{1} R_{2}}{2 B} c_{2}\left(\gamma^{2} \mathrm{E}\left\{s n_{1, r} s n_{\tau, d}\right\}+\mathrm{E}\left\{s n_{1, r} s n_{\tau, t}\right\}+\gamma^{2} \mathrm{E}\left\{s n_{2, r} s n_{\tau, d}\right\}+\mathrm{E}\left\{s n_{2, r} s n_{\tau, t}\right\}\right) \\
& -\gamma \frac{R_{1}^{2}}{2 B} c_{2}\left(\gamma^{2} \mathrm{E}\left\{s n_{1, r} s n_{d}\right\}+\mathrm{E}\left\{s n_{1, r} s n_{t}\right\}+\gamma^{2} \mathrm{E}\left\{s n_{2, r} s n_{d}\right\}+\mathrm{E}\left\{s n_{2, r} s n_{t}\right\}\right) \\
\sigma_{N, s-A S E, 5}^{2}= & \gamma \frac{R_{1} R_{2}}{2 B} c_{1}\left(\gamma^{2} \mathrm{E}\left\{s n_{1, i} s n_{\tau, d}\right\}+\mathrm{E}\left\{s n_{1, i} s n_{\tau, t}\right\}+\gamma^{2} \mathrm{E}\left\{s n_{2, i} s n_{\tau, d}\right\}+\mathrm{E}\left\{s n_{2, i} s n_{\tau, t}\right\}\right) \\
& -\gamma \frac{R_{1}^{2}}{2 B} c_{1}\left(\gamma^{2} \mathrm{E}\left\{s n_{1, i} s n_{d}\right\}+\mathrm{E}\left\{s n_{1, i} s n_{t}\right\}+\gamma^{2} \mathrm{E}\left\{s n_{2, i} s n_{d}\right\}+\mathrm{E}\left\{s n_{2, i} s n_{t}\right\}\right) \\
\sigma_{N, s-A S E, 6}^{2}= & \gamma \frac{R_{2}^{2}}{2 B} c_{2}\left(\gamma^{2} \mathrm{E}\left\{s n_{\tau, 1, r} s n_{\tau, d}\right\}+\mathrm{E}\left\{s n_{\tau, 1, r} s n_{\tau, t}\right\}+\gamma^{2} \mathrm{E}\left\{s n_{\tau, 2, r} s n_{\tau, d}\right\}+\mathrm{E}\left\{s n_{\tau, 2, r} s n_{\tau, t}\right\}\right) \\
- & \gamma \frac{R_{1} R_{2}}{2 B} c_{2}\left(\gamma^{2} \mathrm{E}\left\{s n_{\tau, 1, r} s n_{d}\right\}+\mathrm{E}\left\{s n_{\tau, 1, r} s n_{t}\right\}+\gamma^{2} \mathrm{E}\left\{s n_{\tau, 2, r} s n_{d}\right\}+\mathrm{E}\left\{s n_{\tau, 2, r} s n_{t}\right\}\right) \\
& -\frac{R_{1} R_{2}}{2 B^{2}}\left(\gamma^{4} \mathrm{E}\left\{s n_{d} s n_{\tau, d}\right\}+\gamma^{2} \mathrm{E}\left\{s n_{d} s n_{\tau, t}\right\}+\gamma^{2} \mathrm{E}\left\{s n_{t} s n_{\tau, d}\right\}+\mathrm{E}\left\{s n_{t} s n_{\tau, t}\right\}\right) . \\
\sigma_{N, s-A S E, 7=}^{2} & \gamma \frac{R_{2}^{2}}{2 B} c_{1}\left(\gamma^{2} \mathrm{E}\left\{s n_{\tau, 1, i} s n_{\tau, d}\right\}+\mathrm{E}\left\{s n_{\tau, 1, i} s n_{\tau, t}\right\}+\gamma^{2} \mathrm{E}\left\{s n_{\tau, 2, i} s n_{\tau, d}\right\}+\mathrm{E}\left\{s n_{\tau, 2, i} s n_{\tau, t}\right\}\right) \\
- & \gamma \frac{R_{1} R_{2}}{2 B} c_{1}\left(\gamma^{2} \mathrm{E}\left\{s n_{\tau, 1, i} s n_{d}\right\}+\mathrm{E}\left\{s n_{\tau, 1, i} s n_{t}\right\}+\gamma^{2} \mathrm{E}\left\{s n_{\tau, 2, i} s n_{d}\right\}+\mathrm{E}\left\{s n_{\tau, 2, i} s n_{t}\right\}\right) \\
\sigma_{N, s-A S E, 8}^{2}= & \left.\frac{R_{1}^{2}}{4 B^{2}}\left(\gamma^{4} \mathrm{E}\left\{\left(s n_{d}\right)^{2}\right\}+2 \gamma^{2} \mathrm{E}\left\{s n_{d} s n_{t}\right\}+\mathrm{E}\left\{s n_{t}\right)^{2}\right\}\right) \\
& \left.\left.\left.+s n_{\tau, d}\right)^{2}\right\}+2 \gamma^{2} \mathrm{E}\left\{s n_{\tau, d} s n_{\tau, t}\right\}+\mathrm{E}\left\{\left(s n_{\tau, t}\right)^{2}\right\}\right) \\
& \\
& \\
&
\end{aligned}
$$




\subsection{Contributions to the noise-noise beat variance}

The variance of the noise-noise beat may be separated in several contributions. To illustrate the impact of RX imperfections, the contributions to the noise-noise beat variance of ideal RX, shown in (Costa \& Cartaxo, 2009b), are used as reference. Thus, we find that the $c_{2}^{2}\left(\mathrm{E}\left\{\left(n n_{\|, r}\right)^{2}\right\}-\mathrm{E}^{2}\left\{n n_{\|, r}\right\}+\mathrm{E}\left\{\left(n n_{\perp, r}\right)^{2}\right\}-\mathrm{E}^{2}\left\{n n_{\perp, r}\right\}\right)$ contribution to the noise-noise beat variance results from

$$
\begin{aligned}
\sigma_{N, A S E-A S E, 1}^{2} & =\gamma^{2} \frac{R_{1}^{2}}{4} c_{2}^{2}\left(\mathrm{E}\left\{\left(n n_{\|, r}\right)^{2}\right\}-\mathrm{E}^{2}\left\{n n_{\|, r}\right\}+\mathrm{E}\left\{\left(n n_{\perp, r}\right)^{2}\right\}-\mathrm{E}^{2}\left\{n n_{\perp, r}\right\}\right) \\
& +\gamma^{2} \frac{R_{2}^{2}}{4} c_{2}^{2}\left(\mathrm{E}\left\{\left(n n_{\tau, \|, r}\right)^{2}\right\}-\mathrm{E}^{2}\left\{n n_{\tau, \|, r}\right\}+\mathrm{E}\left\{\left(n n_{\tau, \perp, r}\right)^{2}\right\}-\mathrm{E}^{2}\left\{n n_{\tau, \perp, r}\right\}\right) \\
& +\gamma^{2} \frac{R_{1} R_{2}}{2} c_{2}^{2}\left(\mathrm{E}\left\{n n_{\|, r} n n_{\tau, \|, r}\right\}-\mathrm{E}\left\{n n_{\|, r} \mathrm{E}\left\{n n_{\tau, \|, r}\right\}+\mathrm{E}\left\{n n_{\perp, r} n n_{\tau, \perp, r}\right\}-\mathrm{E}\left\{n n_{\perp, r}\right\} \mathrm{E}\left\{n n_{\tau, \perp, r}\right\}\right)\right.
\end{aligned}
$$

by considering an ideal RX. Similarly, the $c_{1}^{2}\left(E\left\{\left(n n_{\|, i}\right)^{2}\right\}-E^{2}\left\{n n_{\|, i}\right\}+E\left\{\left(n n_{\perp, i}\right)^{2}\right\}-E^{2}\left\{n n_{\perp, i}\right\}\right)$ and $2 c_{1} c_{2}\left(\mathrm{E}\left\{n n_{\|, r} n n_{\|, i}\right\}-\mathrm{E}\left\{n n_{\|, r}\right\} \mathrm{E}\left\{n n_{\|, i}\right\}+\mathrm{E}\left\{n n_{\perp, r} n n_{\perp, i}\right\}-\mathrm{E}\left\{n n_{\perp, r}\right\} \mathrm{E}\left\{n n_{\perp, i}\right\}\right)$ contributions to the noise-noise beat variance result from

$$
\begin{aligned}
\sigma_{N, A S E-A S E, 2}^{2} & =\gamma^{2} \frac{R_{1}^{2}}{4} c_{1}^{2}\left(\mathrm{E}\left\{\left(n n_{\|, i}\right)^{2}\right\}-\mathrm{E}^{2}\left\{n n_{\|, i}\right\}+\mathrm{E}\left\{\left(n n_{\perp, i}\right)^{2}\right\}-\mathrm{E}^{2}\left\{n n_{\perp, i}\right\}\right) \\
& +\gamma^{2} \frac{R_{2}^{2}}{4} c_{1}^{2}\left(\mathrm{E}\left\{\left(n n_{\tau, \|, i}\right)^{2}\right\}-\mathrm{E}^{2}\left\{n n_{\tau, \|, i}\right\}+\mathrm{E}\left\{\left(n n_{\tau, \perp, i}\right)^{2}\right\}-\mathrm{E}^{2}\left\{n n_{\tau, \perp, i}\right\}\right) \\
& +\gamma^{2} \frac{R_{1} R_{2}}{2} c_{1}^{2}\left(\mathrm{E}\left\{n n_{\|, i} n n_{\tau, \|, i}\right\}-\mathrm{E}\left\{n n_{\|, i}\right\} \mathrm{E}\left\{n n_{\tau, \|, i}\right\}+\mathrm{E}\left\{n n_{\perp, i} n n_{\tau, \perp, i}\right\}-\mathrm{E}\left\{n n_{\perp, i}\right\} \mathrm{E}\left\{n n_{\tau, \perp, i}\right\}\right)
\end{aligned}
$$

and

$$
\begin{aligned}
\sigma_{N, A S E-A S E, 3}^{2} & =\gamma^{2} \frac{R_{1}^{2}}{2} c_{1} c_{2}\left(\mathrm{E}\left\{n n_{\|, r} n n_{\|, i}\right\}-\mathrm{E}\left\{n n_{\|, r}\right\} \mathrm{E}\left\{n n_{\|, i}\right\}+\mathrm{E}\left\{n n_{\perp, r} n n_{\perp, i}\right\}-\mathrm{E}\left\{n n_{\perp, r}\right\} \mathrm{E}\left\{n n_{\perp, i}\right\}\right) \\
& +\gamma^{2} \frac{R_{1} R_{2}}{2} c_{1} c_{2}\left(\mathrm{E}\left\{n n_{\tau, \|, r} n n_{\|, i}\right\}-\mathrm{E}\left\{n n_{\tau, \|, r}\right\} \mathrm{E}\left\{n n_{\|, i}\right\}+\mathrm{E}\left\{n n_{\tau, \perp, r} n n_{\perp, i}\right\}-\mathrm{E}\left\{n n_{\tau, \perp, r}\right\} \mathrm{E}\left\{n n_{\perp, i}\right\}\right) \\
& +\gamma^{2} \frac{R_{1} R_{2}}{2} c_{1} c_{2}\left(\mathrm{E}\left\{n n_{\|, r} n n_{\tau, \|, i}\right\}-\mathrm{E}\left\{n n_{\|, r}\right\} \mathrm{E}\left\{n n_{\tau, \|, i}\right\}+\mathrm{E}\left\{n n_{\perp, r} n n_{\tau, \perp, i}\right\}-\mathrm{E}\left\{n n_{\perp, r}\right\} \mathrm{E}\left\{n n_{\tau, \perp, i}\right\}\right) \\
& +\gamma^{2} \frac{R_{2}^{2}}{2} c_{1} c_{2}\left(\mathrm{E}\left\{n n_{\tau, \|, r} n n_{\tau, \|, i}\right\}-\mathrm{E}\left\{n n_{\tau, \|, r}\right\} \mathrm{E}\left\{n n_{\tau, \|, i}\right\}+\mathrm{E}\left\{n n_{\tau, \perp, r} n n_{\tau, \perp, i}\right\}-\mathrm{E}\left\{n n_{\tau, \perp, r}\right\} \mathrm{E}\left\{n n_{\tau, \perp, i}\right\}\right)
\end{aligned}
$$

respectively, when the ideal $R X$ is considered. Other contributions to the noise-noise beat variance arise from the imperfections of the $R X$ and are cancelled when the ideal $R X$ is considered. These contributions are: 
$\sigma_{N, A S E-A S E, 4}^{2}=\gamma \frac{R_{1} R_{2}}{4 B} c_{2}\left(\gamma^{2}\left[\mathrm{E}\left\{n n_{\|, r} n n_{\tau, d, \|,}\right\}-\mathrm{E}\left\{n n_{\|, r}\right\} \mathrm{E}\left\{n n_{\tau, d, d]}\right\}+\mathrm{E}\left\{n n_{\perp, r} n n_{\tau, d, \perp}\right\}-\mathrm{E}\left\{n n_{\perp, r}\right\} \mathrm{E}\left\{n n_{\tau, d, \perp}\right\}\right]\right.$

$\left.\left.+\mathrm{E}\left\{n n_{\|, r} r n_{\tau, t, \|}\right\}\right\}-\mathrm{E}\left\{n n_{\|, r}\right\} \mathrm{E}\left\{n n_{\tau, t, \mid]}\right\}+\mathrm{E}\left\{n n_{\perp, r} n n_{\tau, t, \perp}\right\}-\mathrm{E}\left\{n n_{\perp, r}\right\} \mathrm{E}\left\{n n_{\tau, t, \perp}\right\}\right)$

$-\gamma \frac{R_{1}^{2}}{4 B} c_{2}\left(\gamma^{2}\left[\mathrm{E}\left\{n n_{\|, r} n n_{d, \mid \|}\right\}-\mathrm{E}\left\{n n_{\|, r}\right\} \mathrm{E}\left\{n n_{d, \|}\right\}+\mathrm{E}\left\{n n_{\perp, r} n n_{d, \perp}\right\}-\mathrm{E}\left\{n n_{\perp, r}\right\} \mathrm{E}\left\{n n_{d, \perp}\right\}\right]\right.$

$\left.\left.+\mathrm{E}\left\{n n_{\|, r} n n_{t, \|}\right\}-\mathrm{E}\left\{n n_{\|, r}\right\} \mathrm{E}\left\{n n_{t, \|}\right\}\right\}+\mathrm{E}\left\{n n_{\perp, r} n n_{t, \perp}\right\}-\mathrm{E}\left\{n n_{\perp, r}\right\} \mathrm{E}\left\{n_{t, \perp}\right\}\right)$

$\sigma_{N, A S E-A S E, 5}^{2}=\gamma \frac{R_{1} R_{2}}{4 B} c_{1}\left(\gamma^{2}\left[\mathrm{E}\left\{n n_{\|, i} n n_{\tau, d, \|}\right\}\right]-\mathrm{E}\left\{n n_{\|, i}\right\} \mathbb{E}\left\{n n_{\tau, d, \|}\right\}+\mathrm{E}\left\{n n_{\perp, i} n n_{\tau, d, \perp}\right\}-\mathrm{E}\left\{n n_{\perp, i}\right\} \mathbb{E}\left\{n n_{\tau, d, \perp}\right\}\right]$

$\left.\left.+\mathrm{E}\left\{n n_{\|, i} i n_{\tau, t, \|,}\right\}-\mathrm{E}\left\{n n_{\|, i}\right\} \mathrm{E}\left\{n n_{\tau, t, \|}\right\}\right\}+\mathrm{E}\left\{n n_{\perp, i} n n_{\tau, t, \perp}\right\}-\mathrm{E}\left\{n n_{\perp, i}\right\} \mathrm{E}\left\{n n_{\tau, t, \perp}\right\}\right)$

$-\gamma \frac{R_{1}^{2}}{4 B} c_{1}\left(\gamma^{2}\left[\mathrm{E}\left\{n n_{\|, i} n n_{d, \|}\right\}-\mathrm{E}\left\{n_{\|, i}\right\} \mathrm{E}\left\{n n_{d, \|}\right\}+\mathrm{E}\left\{n n_{\perp, i} i n_{d, \perp}\right\}-\mathrm{E}\left\{n n_{\perp, i}\right\} \mathrm{E}\left\{n n_{d, \perp}\right\}\right]\right.$

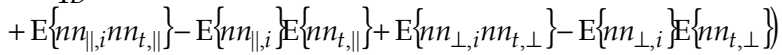

$\sigma_{N, A S E-A S E, 6}^{2}=\gamma \frac{R_{2}^{2}}{4 B} c_{2}\left(\gamma^{2}\left[\mathrm{E}\left\{n n_{\tau, \|, r} n n_{\tau, d, d, \|}\right\}-\mathrm{E}\left\{n n_{\tau, \|, r}\right\} \mathrm{E}\left\{n n_{\tau, d, d]}\right\}+\mathrm{E}\left\{n n_{\tau, \perp, r} n n_{\tau, d, \perp}\right\}-\mathrm{E}\left\{n n_{\tau, \perp, r}\right\} \mathrm{E}\left\{n n_{\tau, d, \perp}\right\}\right]\right.$

$\left.+\mathrm{E}\left\{n n_{\tau, \|, r} n n_{\tau, t, \|, \mid}\right\}-\mathrm{E}\left\{n n_{\tau, \|, r}\right\} \mathrm{E}\left\{n n_{\tau, t, \|,}\right\}+\mathrm{E}\left\{n n_{\tau, \perp, r} n n_{\tau, t, \perp}\right\}-\mathrm{E}\left\{n n_{\tau, \perp, r}\right\} \mathrm{E}\left\{n n_{\tau, t, \perp}\right\}\right)$

$-\gamma \frac{R_{1} R_{2}}{4 B} c_{2}\left(\gamma^{2}\left[\mathrm{E}\left\{n n_{\tau, \|, r} n n_{d, \|}\right\}-\mathrm{E}\left\{n n_{\tau, \|, r}\right\} \mathrm{E}\left\{n n_{d, \|}\right\}+\mathrm{E}\left\{n n_{\tau, \perp, r} n n_{d, \perp}\right\}-\mathrm{E}\left\{n n_{\tau, \perp, r}\right\}\left\{\left[n n_{d, \perp}\right\}\right]\right.\right.$

$\left.+\mathrm{E}\left\{n n_{\tau, \|, r} n n_{t, \|}\right\}-\mathrm{E}\left\{n_{\tau, \|, r}\right\} \mathrm{E}\left\{n n_{t, \|}\right\}+\mathrm{E}\left\{n n_{\tau, \perp, r} n n_{t, \perp}\right\}-\mathrm{E}\left\{n n_{\tau, \perp, r}\right\} \mathrm{E}\left\{n n_{t, \perp}\right\}\right)$

$$
\begin{aligned}
& \left.\sigma_{N, A S E-A S E, 7}^{2}=\frac{R_{1}^{2}}{16 B^{2}}\left(\gamma^{4}\left[\mathrm{E}\left\{\left(n n_{d, 1}\right)\right)^{2}\right\}-\mathrm{E}^{2}\left\{n n_{d, \mid}\right\}\right\}+\mathrm{E}\left\{\left(n n_{d, \perp}\right)^{2}\right\}-\mathrm{E}^{2}\left\{n n_{d, \perp}\right]\right] \\
& \left.\left.\left.+2 \gamma^{2}\left[\mathrm{E}\left\{n n_{d, \mid} \mid n n_{t, 1}\right\}\right\}-\mathrm{E}\left\{n n_{d, \mid}\right\}\right] \mathbb{E}\left\{n n_{t, \mid}\right\}\right\}+\mathrm{E}\left\{n n_{d, \perp} n n_{t, \perp}\right\}-\mathrm{E}\left\{n n_{d, \perp}\right\} \mathbb{E}\left\{n n_{t, \perp}\right\}\right] \\
& \left.+\mathrm{E}\left\{\left(n n_{t, 1}\right)^{2}\right\}-\mathrm{E}^{2}\left\{n n_{t, 1}\right\}+\mathrm{E}\left\{\left(n n_{t, \perp}\right)^{2}\right\}-\mathrm{E}^{2}\left\{n n_{t, \perp}\right\}\right) \\
& \left.+\frac{R_{2}^{2}}{16 B^{2}}\left(\gamma^{4}\left[\mathrm{E}\left\{\left(n n_{\tau, d, 1}\right)\right)^{2}\right\}-\mathrm{E}^{2}\left\{n n_{\tau, d, \mid}\right\}\right\}+\mathrm{E}\left\{\left(n n_{\tau, d, \perp}\right)^{2}\right\}-\mathrm{E}^{2}\left\{n n_{\tau, d, \perp}\right\}\right] \\
& \left.+2 \gamma^{2}\left[\mathrm{E}\left\{n n_{\tau, d, \mid} \mid n n_{\tau, t, \mid}\right\}\right\}-\mathrm{E}\left\{n n_{\tau, d, \mid}\right\} \mathrm{E}\left\{n n_{\tau, t,|,|}\right\}+\mathrm{E}\left\{n n_{\tau, d, \perp} n n_{\tau, t, \perp}\right\}-\mathrm{E}\left\{n n_{\tau, d, \perp}\right\} \mathrm{E}\left\{n n_{\tau, t, \perp}\right\}\right] \\
& \left.\left.+\mathrm{E}\left\{\left(n n_{\tau, t, \mid}\right)^{2}\right\}-\mathrm{E}^{2}\left\{n n_{\tau, t, \mid}\right\}\right\}+\mathrm{E}\left\{\left(n n_{\tau, t, \perp}\right)^{2}\right\}-\mathrm{E}^{2}\left\{n n_{\tau, t, \perp}\right\}\right) \\
& \left.-\frac{R_{1} R_{2}}{8 B^{2}}\left(\gamma^{4}\left[\mathrm{E}\left\{n n_{d, \mid} \mid n n_{\tau, d, \mid}\right\}\right\}-\mathrm{E}\left\{n n_{d, \mid}\right\} \in \mathbb{E}\left\{n n_{\tau, d, \mid}\right\}\right\}+\mathrm{E}\left\{n n_{d, \perp} n n_{\tau, d, \perp}\right\}-\mathrm{E}\left\{n n_{d, \perp}\right\} \mathbb{E}\left\{n n_{\tau, d, \perp}\right\}\right] \\
& \left.+\gamma^{2}\left[\mathrm{E}\left\{n n_{d, \mid}\left|n n_{\tau, t, \mid}\right|\right\}-\mathrm{E}\left\{n_{d, \mid} \mid\right\} E\left\{n n_{\tau, t, \mid}\right\}\right\}+\mathrm{E}\left\{n n_{d, \perp} n n_{\tau, t, \perp}\right\}-\mathrm{E}\left\{n n_{d, \perp}\right\} \mathbb{E}\left\{n n_{\tau, t, \perp}\right\}\right] \\
& \left.\left.+\gamma^{2}\left[\mathrm{E}\left\{n n_{t, \mid} \mid \eta n_{\tau, d, \mid}\right\}\right\}-\mathrm{E}\left\{n_{t,|,|}\right\} \mathrm{E}\left\{n n_{\tau, d, \mid}\right\}\right\}+\mathrm{E}\left\{n n_{t, \perp} n n_{\tau, d, \perp}\right\}-\mathrm{E}\left\{n n_{t, \perp}\right\} \mathbb{E}\left\{n n_{\tau, d, \perp}\right\}\right] \\
& \left.\left.\left.+\mathrm{E}\left\{n n_{t, \mid} \mid \eta n_{\tau, t, \mid}\right\}-\mathrm{E}\left\{n n_{t, \mid}\right]\right\} \mathbb{E}\left\{n n_{\tau, t, \mid}\right\}\right\}+\mathrm{E}\left\{n n_{t, \perp} n n_{\tau, t, \perp}\right\}-\mathrm{E}\left\{n n_{t, \perp}\right\} \mathbb{E}\left\{n n_{\tau, t, \perp}\right\}\right)
\end{aligned}
$$




$$
\begin{aligned}
& \sigma_{N, A S E-A S E, 8}^{2}=\gamma \frac{R_{2}^{2}}{4 B} c_{1}\left(\gamma^{2}\left[\mathrm{E}\left\{n n_{\tau, \mid, i} n n_{\tau, d, \mid}\right\}-\mathrm{E}\left\{n n_{\tau, \mid, j}\right\} \mathrm{E}\left\{n n_{\tau, d, \mid}\right\}\right]+\mathrm{E}\left\{n n_{\tau, \perp, i} n n_{\tau, d, \perp}\right\}-\mathrm{E}\left\{n n_{\tau, \perp, i}\right] \mathrm{E}\left\{n n_{\tau, d, \perp}\right\}\right] \\
& \left.\left.+\mathrm{E}\left\{n n_{\tau, \mid, i} n n_{\tau, t, \mid}\right\}\right\} \mathrm{E}\left\{n n_{\tau,|,|}\right\} \mathrm{E}\left\{n n_{\tau, t, \mid}\right\}+\mathrm{E}\left\{n n_{\tau, \perp, i} n n_{\tau, t, \perp}\right\}-\mathrm{E}\left\{n n_{\tau, \perp, i}\right\} \mathrm{E}\left\{n n_{\tau, t, \perp}\right\}\right) \\
& \left.-\gamma \frac{R_{1} R_{2}}{4 B} c_{1}\left(\gamma^{2}\left[\mathrm{E}\left\{n n_{\tau,||, i} n n_{d, \mid}\right\}\right]-\mathrm{E}\left\{n n_{\tau, \mid}\right\}, \mathrm{E}\left\{n n_{d, \mid}\right\}\right]+\mathrm{E}\left\{n n_{\tau, \perp, i} n n_{d, \perp}\right\}-\mathrm{E}\left\{n n_{\tau, \perp, i}\right\} \mathrm{E}\left\{n n_{d, \perp}\right\}\right] \\
& \left.\left.+\mathrm{E}\left\{n n_{\tau,|,|} n n_{t, \mid}\right\}\right\} \mathrm{E}\left\{n n_{\tau, \mid,}\right\} \mathrm{E}\left\{n n_{t,||}\right\}+\mathrm{E}\left\{n n_{\tau, \perp, i} n n_{t, \perp}\right\}-\mathrm{E}\left\{n n_{\tau, \perp, i}\right\} \mathrm{E}\left\{n n_{t, \perp}\right\}\right)
\end{aligned}
$$

\subsection{List of acronyms}

AWGN - Additive white Gaussian noise

BEP - Bit error probability

DCF - Dispersion compensating fiber

DPSK - Differential phase-shift-keying

DQPSK - Differential quadrature phase-shift-keying

EDFA - Erbium doped fiber amplifier

EDP- Equivalent differential phase

GA- Gaussian approximation

GVD - Group velocity dispersion

I - In-phase

MC - Monte-Carlo

MZDI - Mach-Zehnder delay interferometer

NRZ - Non-return-to-zero

OOK - On-off keying

OSNR - Optical signal-to-noise ratio

PDF - Probability density function

PIN - Positive-intrinsic-negative photodetector

PSD - Power spectral density

Q - Quadrature

RX - Receiver

RZ - Return-to-zero

SASM - Semi-analytical simulation method

SPM - Self-phase modulation

SSMF - Standard single-mode fiber

STD - Standard deviation

TX - Transmitter 


\section{References}

Bosco, G. \& Poggiolini, P. (2006). On the joint effect of receiver impairments on directdetection DQPSK systems, IEEE/OSA J. Lightwave Technol., vol. 24, no. 3, Mar. 2006, pp. 1323-1333.

Carena, C., Curri, V. et al. (1998). On the joint effects of fiber parametric gain and birefringence and their influence on ASE noise, IEEE/OSA J. Lightwave Technol., vol. 16, no. 7, Jul. 1998, pp. 1149-1157.

Costa, N. \& Cartaxo, A. (2007). BER estimation in DPSK systems using the differential phase $\mathrm{Q}$ taking into account the electrical filtering influence, IEEE Proc. Intern. Microwave and Optoelectron. Conf., Salvador, Brazil, Oct. 2007, pp. 337-340.

Costa, N. \& Cartaxo, A. (2009). Novel semi-analytical method for BER evaluation in simulated optical DQPSK systems, IEEE Photon. Technol. Lett., vol. 21, no. 7, Apr. 2009, pp. 447-449.

Costa, N. \& Cartaxo, A. (2009b). Optical DQPSK system performance evaluation using equivalent differential phase in presence of receiver imperfections, IEEE/OSA J. Lightwave Technol., submitted paper.

Demir, A. (2007). Nonlinear phase noise in optical-fiber-communication systems, J. Lightwave Technol., vol. 25, no. 8, Aug. 2007, pp. 2002-2032.

Hanik, N. (2002). Modelling of nonlinear optical wave propagation including linear modecoupling and birefringence, Optics Communications, vol. 214, Dec. 2002, pp. 207-230.

Ho, K.-P. (2005). Phase-Modulated Optical Communication Systems, Springer, ISBN-13: 978-0387-24392-4, United States of America, 2005.

Ip, E. \& Kahn, J. (2006). Power spectra of return-to-zero optical signals, IEEE/OSA J. Lightwave Technol., vol. 24, no. 3, Mar. 2006, pp. 1610-1618.

Iannone, E., Matera, F. et al. (1998). Nonlinear Optical Communication Networks, John Wiley \& Sons, ISBN: 0-471-15270-6, United States of America, 1998.

Jeruchim, M., Balaban, P. \& Shanmugan, K. (2000). Simulation of Communication Systems Modelling, Methodology and Techniques, Kluwer Academic/Plenum Publishers, ISBN: 0-306-46267-2, United States of America, 2000.

Marcuse, D., Menyuk, C. R. \& Way, P. K. (1997). Application of the Manakov-PMD equation to studies of signal propagation in optical fibers with randomly varying birefringence, IEEE/OSA J. Lightwave Technol., vol. 15, no. 9, Sept. 1997, pp. 17351746.

Morita, I. \& Yoshikane, N. (2005). Merits of DQPSK for ultrahigh capacity transmission, Proc. IEEE LEOS Annual Meeting, Sydney, Australia, Oct. 2005, paper We5.

Rebola, J. L. \& Cartaxo, A. (2001). Gaussian approach for performance evaluation of optically preamplified receivers with arbitrary optical and electrical filters, IEE Proc. Optoelectron., vol. 148, no. 3, Jun. 2001, pp. 135-142.

Winzer, P. J. \& Essiambre, R.-J. (2006). Advanced modulation formats for high-capacity optical transport networks, IEEE/OSA J. Lightwave Technol., vol. 24, no. 12, Dec. 2006, pp. 4711-4728.

Winzer, P. J., Raybon, G. et al. (2008). 100-Gb/s DQPSK transmission: from laboratory experiments to field trials, IEEE/OSA J. Lightwave Technol., vol. 26, no. 20, Oct. 2008, pp. 3388-3402. 
Xu, C., Liu, X. \& Wei, X. (2004). Differential phase-shift keying for high spectral efficiency optical transmissions, IEEE J. Select. Topics in Quantum Electron., vol. 10, no. 2, Mar./Apr. 2004, pp. 281-293. 


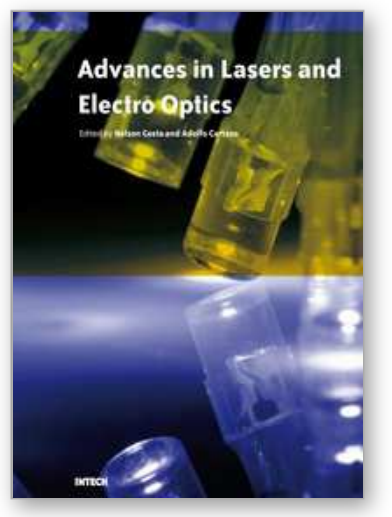

\author{
Advances in Lasers and Electro Optics \\ Edited by Nelson Costa and Adolfo Cartaxo
}

ISBN 978-953-307-088-9

Hard cover, 838 pages

Publisher InTech

Published online 01, April, 2010

Published in print edition April, 2010

Lasers and electro-optics is a field of research leading to constant breakthroughs. Indeed, tremendous advances have occurred in optical components and systems since the invention of laser in the late $50 \mathrm{~s}$, with applications in almost every imaginable field of science including control, astronomy, medicine, communications, measurements, etc. If we focus on lasers, for example, we find applications in quite different areas. We find lasers, for instance, in industry, emitting power level of several tens of kilowatts for welding and cutting; in medical applications, emitting power levels from few milliwatt to tens of Watt for various types of surgeries; and in optical fibre telecommunication systems, emitting power levels of the order of one milliwatt. This book is divided in four sections. The book presents several physical effects and properties of materials used in lasers and electro-optics in the first chapter and, in the three remaining chapters, applications of lasers and electro-optics in three different areas are presented.

\title{
How to reference
}

In order to correctly reference this scholarly work, feel free to copy and paste the following:

Nelson Costa and Adolfo Cartaxo (2010). Optical DQPSK Modulation Performance Evaluation, Advances in Lasers and Electro Optics, Nelson Costa and Adolfo Cartaxo (Ed.), ISBN: 978-953-307-088-9, InTech, Available from: http://www.intechopen.com/books/advances-in-lasers-and-electro-optics/optical-dqpskmodulation-performance-evaluation

\section{INTECH}

open science | open minds

\section{InTech Europe}

University Campus STeP Ri

Slavka Krautzeka 83/A

51000 Rijeka, Croatia

Phone: +385 (51) 770447

Fax: +385 (51) 686166

www.intechopen.com

\section{InTech China}

Unit 405, Office Block, Hotel Equatorial Shanghai

No.65, Yan An Road (West), Shanghai, 200040, China

中国上海市延安西路65号上海国际贵都大饭店办公楼 405 单元

Phone: +86-21-62489820

Fax: $+86-21-62489821$ 
(C) 2010 The Author(s). Licensee IntechOpen. This chapter is distributed under the terms of the Creative Commons Attribution-NonCommercialShareAlike-3.0 License, which permits use, distribution and reproduction for non-commercial purposes, provided the original is properly cited and derivative works building on this content are distributed under the same license. 